\title{
Consensus-based technical recommendations for clinical translation of renal diffusion-weighted MRI
}

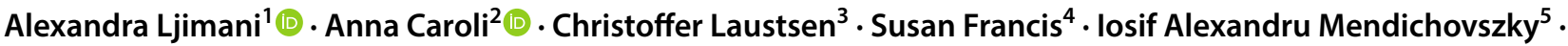 \\ Octavia Bane $^{6} \cdot$ Fabio Nery $^{7} \cdot$ Kanishka Sharma $^{8} \cdot$ Andreas Pohlmann $^{9} \cdot$ Ilona A. Dekkers $^{10} \cdot$ Jean-Paul Vallee ${ }^{11}$. \\ Katja Derlin ${ }^{12}$. Mike Notohamiprodjo ${ }^{13,26} \cdot$ Ruth P. Lim $^{14}$. Stefano Palmucci ${ }^{15}$. Suraj D. Serai ${ }^{16}$. Joao Periquito ${ }^{9}$. \\ Zhen Jane Wang ${ }^{17} \cdot$ Martijn Froeling ${ }^{18}$ (1) Harriet C. Thoeny ${ }^{19} \cdot$ Pottumarthi Prasad $^{20} \cdot$ Moritz Schneider $^{21,22}$. \\ Thoralf Niendorf $^{9} \cdot$ Pim Pullens $s^{23,24} \cdot$ Steven Sourbron ${ }^{8} \cdot$ Eric E. Sigmund $^{25}$
}

Received: 6 August 2019 / Revised: 17 October 2019 / Accepted: 19 October 2019 / Published online: 1 November 2019

(c) The Author(s) 2019, corrected publication 2020

\begin{abstract}
Objectives Standardization is an important milestone in the validation of DWI-based parameters as imaging biomarkers for renal disease. Here, we propose technical recommendations on three variants of renal DWI, monoexponential DWI, IVIM and DTI, as well as associated MRI biomarkers (ADC, $D, D^{*}, f, \mathrm{FA}$ and $\mathrm{MD}$ ) to aid ongoing international efforts on methodological harmonization.

Materials and methods Reported DWI biomarkers from 194 prior renal DWI studies were extracted and Pearson correlations between diffusion biomarkers and protocol parameters were computed. Based on the literature review, surveys were designed for the consensus building. Survey data were collected via Delphi consensus process on renal DWI preparation, acquisition, analysis, and reporting. Consensus was defined as $\geq 75 \%$ agreement.

Results Correlations were observed between reported diffusion biomarkers and protocol parameters. Out of 87 survey questions, 57 achieved consensus resolution, while many of the remaining questions were resolved by preference (65-74\% agreement). Summary of the literature and survey data as well as recommendations for the preparation, acquisition, processing and reporting of renal DWI were provided.

Discussion The consensus-based technical recommendations for renal DWI aim to facilitate inter-site harmonization and increase clinical impact of the technique on a larger scale by setting a framework for acquisition protocols for future renal DWI studies. We anticipate an iterative process with continuous updating of the recommendations according to progress in the field.
\end{abstract}

Keywords Biomarker · DWI · ADC · IVIM · DTI

\section{Introduction}

Diffusion-weighted (DWI) magnetic resonance imaging (MRI) has been shown to provide differentiated information on the microstructure of kidney tissue. Furthermore, significant efforts have been made to adopt DWI-based parameters as an MR biomarker for functional renal

Electronic supplementary material The online version of this article (https://doi.org/10.1007/s10334-019-00790-y) contains supplementary material, which is available to authorized users.

Alexandra Ljimani

alexandra.ljimani@med.uni-duesseldorf.de

Extended author information available on the last page of the article imaging [1-6]. However, to successfully translate the research results of renal DWI to clinical practice, there are still some challenges to overcome. Firstly, acquisition protocols vary between research groups and reflect local practice and expertise. Secondly, patient preparation, data post-processing and image analysis are not standardized, with several approaches being used by different research groups. As has been recognized by other consortium efforts [7-9], our motivation behind prioritizing standardization of these processes is the generation of reliable MRI biomarkers that are ready to be broadly utilized in multi-site studies. When achieved, the data generated from standardized study protocols will sufficiently increase the evidence base to determine threshold values 
for DWI-based parameters, to differentiate between renal pathologies. Histopathological correlation should also continue to be performed to ensure diagnostic validation of the MRI biomarkers. With the aim to move toward a standardization and to facilitate the validation of DWIbased parameters as a renal MRI biomarker, an international, multidisciplinary group of renal imaging researchers with experience and/or ongoing work in renal DWI was recently formed as part of the 'PARENCHIMA' (the European Cooperation in Science and Technology) COST action (www.renalmri.org).

As a first step in this endeavour, Caroli et al. [10] published a review and statement paper reflecting the current state of research to assess diffuse renal pathology by renal DWI. The work summarizes the acquisition protocols used in human renal DWI studies up to August 2017 (172 studies) involving both healthy subjects and patients with renal disease. It highlights the large diversity in acquisition protocols, patient preparation and image post-processing techniques, as well as the lack of "gold standard" for the measurement of in vivo renal DWI. This diversity of acquisition protocols across studies has led to a variability of acquired quantitative renal diffusion parameters, which is summarized in the detailed supplement material of the review [10]. Therefore, a further mission of the PARENCHIMA initiative is building consensus on renal DWI acquisition protocol, patient preparation and postprocessing techniques.

In this work, a consensus on recommended acquisition protocol for renal DWI was formed consistent with the consensus-building goals of the Delphi process [11-13]. The design of the surveys for the consensus building was informed by a literature review (extending the prior review until November 2018) that aimed to identify which acquisition parameters had the most impact on DWI measurements. For the development of the recommendations, the three most common variants of renal DWI techniques used in the literature were considered: (1) monoexponential model with parameter apparent diffusion coefficient (ADC); (2) biexponential model or IVIM (intravoxel incoherent motion) model with the parameters water diffusion in the tissue $(D)$, flowing fraction $(f)$ and pseudodiffusion $\left(D^{*}\right)$; and (3) diffusion tensor imaging (DTI) with mean diffusivity (MD) and fractional anisotropy (FA). All three variants of renal DWI techniques aim to estimate a diffusion constant of water in tissue. However, in all models this diffusion constant is named differently (ADC, $D$, and MD). ADC quantification methods considering a non-Gaussian DWI signal behavior are not covered in these recommendations given their more preliminary stage of investigation and are not deemed as ripe for standardization as the other methods described above. We summarize the three common renal DWI approaches and associated quantification methods below.

\section{Monoexponential ADC}

This quantification model for diffusion-weighted MRI is the most popular due to its simplicity and modest acquisition requirements. The monoexponential ADC model assumes a uniform Gaussian displacement distribution of the water molecules corresponding to a monoexponential diffusion-weighted signal decay of the MR signal. The computation of the monoexponential ADC is based on the Stejskal-Tanner equation [14]:

$\frac{S_{\mathrm{b}}}{S_{0}}=e^{-b \mathrm{ADC}_{\text {mono }}}$

where $S_{\mathrm{b}}$ is the diffusion-weighted signal intensity, $S_{0}$ is the signal intensity without a diffusion weighting $(b=0 \mathrm{~s} /$ $\mathrm{mm}^{2}$ ), $b$ is the diffusion weighting strength (in $\mathrm{s} / \mathrm{mm}^{2}$ ), and $\mathrm{ADC}_{\text {mono }}$ is the apparent diffusion coefficient of water within the observed image voxel.

For renal tissue, the monoexponential model is known to be insufficient to describe the diffusion-weighted signal decay, with IVIM effects occurring at low $b$ values $\left(<200 \mathrm{~s} / \mathrm{mm}^{2}\right)[15]$ and non-Gaussian effects possibly occurring at high $b$ values $\left(>800 \mathrm{~s} / \mathrm{mm}^{2}\right)$. However, as a single parameter estimation, the monoexponential model provides relatively robust $\mathrm{ADC}$ and requires only moderate signal-to-noise ratio on DWI.

Given the contrast effects mentioned above, the estimated ADC is strongly dependent on the choice of selected b-values $[15,16]$ and no consensus exists with regard to the choice and number of $b$ values in a renal DWI acquisition protocol. Taking into account Eq. (1), a set of minimum two $b$ values is enough to reach a stable diffusion signal $[16,17]$ for the quantification of ADC. However, most authors prefer to describe the diffusion signal decay more precisely by including more $b$ values in the acquisition protocol. Considering possible anisotropic diffusion, it is common practice to measure the $b$ values in several orthogonal directions during the ADC acquisition [15, 16].

\section{Intravoxel incoherent motion (IVIM)}

First described by Le Bihan et al. [18] in 1986 the IVIM model is another option to interpret the physiological underpinning of the diffusion signal. Since the initial studies in human subjects by Muller et al. in 1998 [19] and later by Thoeny et al. in 2006 [20] showing the potential of the IVIM model to interpret diffusion signal in the kidney, this quantification has been demonstrated to improve the representation of the diffusion-weighted signal in renal tissue compared to the ADC [21-23]. 
IVIM considers the diffusion signal originating from two different compartments. One compartment reflects the slow thermal diffusion in the tissue $(D)$, hindered or restricted by local microstructure. The second compartment considers the fast molecule movement associated with incoherent flow in the microvasculature or renal tubules that mimic random water motion assuming that many vessel and tubules orientations are present within the voxel (quantified by the pseudodiffusion, $D^{*}$ and the flowing fraction, $f$ ).

This method of quantification utilizes a biexponential decay, describing the overall diffusion-weighted signal as the sum of the diffusion and flowing components:

$\frac{S_{\mathrm{b}}}{S_{0}}=(1-f) e^{-b D}+f e^{-b D^{*}}$

where $S_{\mathrm{b}}$ is the diffusion-weighted signal intensity, $S_{0}$ is the signal intensity without a diffusion weighting ( $b=0 \mathrm{~s} /$ $\left.\mathrm{mm}^{2}\right), b$ is the diffusion weighting strength (in $\mathrm{s} / \mathrm{mm}^{2}$ ), $D$ is the water diffusion in the tissue (slow component), $D^{*}$ is the pseudodiffusion (fast component), and $f$ is the flowing fraction.

To quantify IVIM parameters, a minimum of four $b$ values are needed to determine all unknown parameters in Eq. (2), which typically extends the acquisition time in comparison to the monoexponential ADC. Furthermore, there is no universally accepted algorithm yet to calculate IVIM quantitative parameters. In many studies, a so-called "segmented fitting" or "2-step" approach is used to calculate the IVIM parameters (2), due to its extended stability and faster fitting [24-27]. In the "segmented fitting", a threshold $b$ value is defined to separate flowing from diffusion effects (microcirculation-induced decay assumed negligible above this threshold). However, although $D$ is more stable in the "segmented fitting", than in others, the estimates of $f$ and $D^{*}$ can be biased depending on threshold choice. More recently, Bayesian probability-based fitting methods have been explored, with or without fixing of the pseudodiffusion coefficient (this has shown higher precision/accuracy, and low inter-subject variability [28]).

Other, more complex, extended IVIM models can be found in the literature that aim to incorporate more characteristics of functioning renal tissue into the signal description. Three compartment models include an additional component taking into account multiple sources of intravoxel incoherent motion, e.g., due to the glomerular flow [29, 30], vascular vs. tubular flow, or residual fat signal [31]. Other extended models combine IVIM with diffusion anisotropy for a more comprehensive description of both structural and microcirculation features [32, 33]. These models are mentioned here solely to indicate current research frontiers as they require further investigation before they can be pursued in the context of consensus standardization.

\section{Diffusion tensor imaging (DTI)}

Measurement of the directional dependence (anisotropy) of apparent diffusion in tissue microstructure provides a marker of that tissue's integrity and thereby its clinical function. Diffusion tensor imaging (DTI) quantitatively measures and maps the anisotropy imposed on water diffusion by a tissue's microstructure.

For DTI analysis, diffusion-weighted signals along several diffusion directions are acquired and fit to a $3 \times 3$ symmetric tensor model [34, 35].

$\bar{D}=\left(\begin{array}{lll}D_{x x} & D_{x y} & D_{x z} \\ D_{y x} & D_{y y} & D_{y z} \\ D_{z x} & D_{z y} & D_{z z}\end{array}\right)$

where $\bar{D}$ is the symmetric diffusion tensor with elements $D_{\mathrm{ij}}$ determined by the linear set of equations generated by the set of ADC measurements along each diffusion gradient direction [36] $\hat{g}$ :

$\mathrm{ADC}_{n}={\widehat{g_{n}}}^{\dagger} \cdot \bar{D} \cdot \widehat{g_{n}}=\sum_{i j} g_{i, n} g_{j, n} D_{i j}$.

More generally, all gradients (imaging and diffusion weighting) can be taken into account by computing the full b-matrix of their diffusion weighting:

$-\ln \left(\frac{S}{S_{0}}\right)=\sum_{i j} b_{i j} D_{i j}$

$b_{i j}=\int_{0}^{\mathrm{TE}} k_{i}(t) k_{j}(t) d t$,
$k_{i}(t)=\int_{0}^{t} \gamma G_{i}\left(t^{\prime}\right) d t^{\prime}$.

The eigenvalues of this tensor describe the maximal, intermediate, and minimal diffusion values, with eigenvectors reflecting their corresponding orientation. The primary eigenvector, associated with the largest eigenvalue, indicates the orientation of maximal diffusion.

$\mathrm{MD}=\frac{1}{3}\left(\lambda_{1}+\lambda_{2}+\lambda_{3}\right)$

where MD or mean diffusivity is the average of the diffusion coefficients and $\lambda_{1}, \lambda_{2}, \lambda_{3}$ are the eigenvalues. 
Another parameter, called fractional anisotropy (FA), reflects the amount of diffusion directivity in DTI studies $(0=$ complete isotropy, $1=$ complete anisotropy) and is calculated by

$\mathrm{FA}=\sqrt{\frac{3}{2}} \times \frac{\sqrt{\left(\lambda_{1}-\overline{M D}\right)^{2}+\left(\lambda_{2}-\overline{M D}\right)^{2}+\left(\lambda_{3}-\overline{M D}\right)^{2}}}{\sqrt{\lambda_{1}^{2}+\lambda_{2}^{2}+\lambda_{3}^{2}}}$,

where again $\overline{\mathrm{MD}}$ is the average of the diffusion coefficients, a DTI-specific ADC equivalent and $\lambda_{1}, \lambda_{2}, \lambda_{3}$ are the eigenvalues.

Several studies have demonstrated that DTI provides powerful biomarkers of diffusion isotropy in the cortex and anisotropy in the renal medulla [15, 16, 22, 37-43]. This behavior is consistent with the known structural organization of medullary constituents such as the tubular loops of Henle, collecting ducts, and vascular vasa recta, which have an inward radial pattern toward the renal pelvis.

As with many diffusion biomarkers, FA and MD depend on the number and magnitude of the applied $b$ values [38, 42, 44]. As diffusion anisotropy is a key target of DTI, acquisition of multiple diffusion directions (minimally 6) is required for tensor computation. However, while some studies of diffusion direction choice in renal DTI have been performed supporting at least 12 directions [45], determination of an optimal number or choice of $b$ values and directions for renal DTI, analogous to comprehensive efforts in the brain [28] or muscle [46], has not yet been performed.

\section{Materials and methods}

\section{Literature review and data extraction}

To justify the motivation for the standardization process, assess the state of the renal DWI literature, and provide input to subsequent recommendations, we summarize reported DWI biomarkers from a wide range of prior renal DWI studies assessing diffuse renal diseases. These efforts build upon reviews and meta-analyses that have aimed to understand the variability of reported renal diffusion biomarkers in the literature $[4,15,23]$.

A systematic review and analysis of the literature (using the same search criteria in PubMed as previously used by Caroli et al. [10], but extending those until November 2018) was carried out.

Specifically, papers were categorized according to their protocol and quantification scheme as either monoexponential DWI (113 studies), DTI (40 studies), or IVIM (41 studies). From each paper, we extracted protocol parameters including full $b$ value ranges, repetition times (TR), echo times (TE), number of gradient directions and field strength. The distribution of $b$ value ranges was extracted for each DWI model for visualization. Additionally, DWI biomarkers were also extracted for cortex, medulla, and whole kidney (as available in each study), reporting values in healthy adult controls. For each $b$ value range, the maximum and average $b$ values were also computed. Monoexponential DWI studies provided ADC values [20, 21, 43, 47-61], DTI studies provided MD and FA values [29, 32, 33, 38, 42, 45, 62-71] and IVIM studies provided $D, f$ and $D^{*}$ values [20, 21, 28, 29, 32, 42, 43, 54, 55, 68, 71-78].

Following data extraction, correlations were computed in healthy volunteers only via Pearson correlation coefficients with the following protocol parameters: (1) TR; (2) TE; (3) average $b$ value; (4) maximum $b$ value; (5) transverse relaxation factor $\mathrm{T} 2=\exp (-\mathrm{TE} / \mathrm{T} 2 t) ;(6) \mathrm{T} 1=[1-\exp (-\mathrm{TR} /$ $\mathrm{T} 1 t)]$, where $\mathrm{T} 2 t=87 \mathrm{~ms}$ and $\mathrm{T} 1 t=1147 \mathrm{~ms}$ were taken as representative relaxation times for renal tissue at 3.0 $\mathrm{T}$ [79]. After correlation with individual protocol parameters, correlations were computed between diffusion biomarkers and all possible products or ratios of the protocol parameters (52 combinations in all). Correlation coefficients $R$ and significance levels $p$ were derived for each correlation using the Igor Pro 7 software (Wavemetrics, Inc., Lake Oswego, OR USA). Significant correlations were noted both without $(p<0.05)$ and with Bonferroni correction for multiple comparisons $(p<0.05 / 52=0.00096)$. Finally, all diffusion biomarkers from healthy volunteers were grouped according to field strength $(1.5 \mathrm{~T}$ or $3.0 \mathrm{~T})$ and compared for differences with a two-tailed Student's $t$ test, for which significant differences are indicated for $p<0.05$.

\section{Description of survey process}

As described in the accompanying covering letter by Sourbron et al. and in keeping with the 'approximation of a twostep modified Delphi method [80]' for consensus building, a survey was circulated using a publicly available tool (Google Forms) to a range of renal imaging researchers with experience and/or ongoing activity in renal diffusion imaging. In addition to offering participation to all members of the PARENCHIMA collaboration, every effort was made to invite at least one researcher or corresponding author from each group contributing to the literature as surveyed previously [10]. Two rounds of surveys were circulated over a period of 4 months. Between the first and second circulation and following review of initial results with the ASL, BOLD and T1/T2 panels at a meeting in Aarhus, the list of questions was increased and refined to avoid ambiguity and increase the likelihood of reaching consensus on as many items as possible. The surveys included questions on: respondent training, patient preparation, image acquisition, diffusion parameters, analysis, and reporting. The full list of 
questions from the final circulation is provided in "Results" along with summarized results, percentage agreement, no basis and disagreement for all responses, as well as percentage agreement and disagreement without abstentions. Nearly, all questions tested level of agreement or disagreement qualitatively. In the first circulation, five options were provided (strongly agree/agree/neutral/disagree/strongly disagree). In the second circulation, the available responses were simplified and allowed for abstention (agree/disagree/I have insufficient experience to make a recommendation). Other questions focused on the preferred field strength or allowed multiple selections to test support of multiple related issues (e.g., reported parameters). Text comments were also collected on sets of questions of similar topics. For both rounds, responses were aggregated following the completion of the survey. The first round survey was issued on 11 January 2019, and the second on 27 March 2019; both were open for approximately 1 month.

After excluding abstentions, the level of agreement or disagreement as a percentage of all responses was calculated for each question. Responses for which either agreement or disagreement reached $75 \%$ or higher were deemed to have achieved consensus. Responses that related to one that has already reached consensus were deemed to have been resolved. For responses with agreement levels between 60 and 75\%, a 'preference' was indicated but without the full weight of consensus. Similarly, other responses mutually exclusive from a preference or reaching lower levels of agreement on the same topic were deemed to have been resolved by that preference. Finally, the combination of all of these directly or indirectly
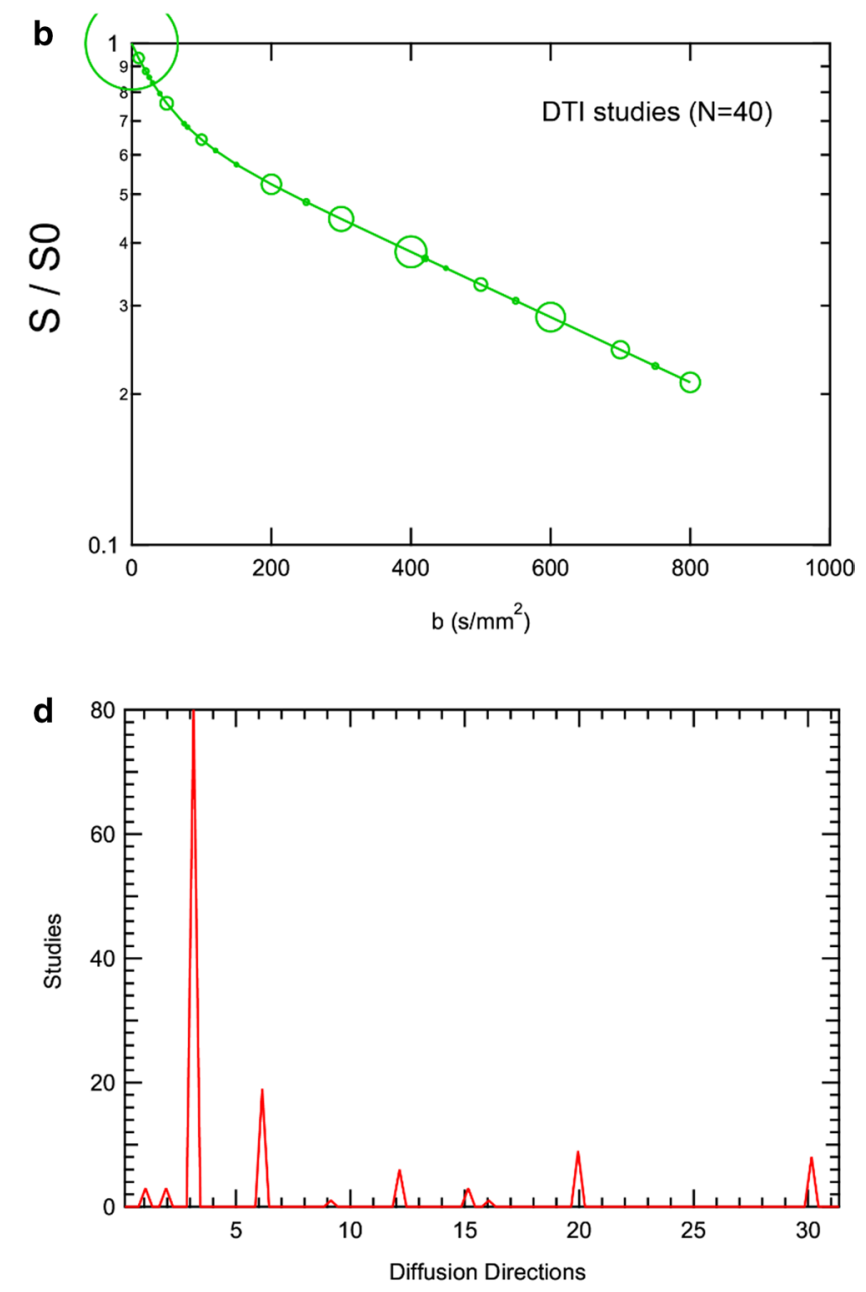

d Distribution of diffusion directions employed; ADC and IVIM studies dominantly employed three directions, with DTI studies employing more directions 
resolved questions was considered to generate a set of recommendations.

\section{Results}

\section{Literature review}

Figure 1 shows the distributions of $b$ value sampling and diffusion directions from all renal studies considered (control and patient related). Monoexponential DWI and IVIM studies have featured a continuous range of $b$ values, while DTI studies have used a sparser selection, consistent with more time devoted to directional sampling. Finally, the majority of ADC and IVIM studies used three orthogonal directions for isotropic imaging. Since many of these studies employ inline processing with vendor software, the three directions are typically immediately averaged, both for convenience and for enhanced signal-to-noise ratio, to generate approximate 'trace-weighted' images prior to generation of ADC maps. DTI studies used six directions most often, but studies using as many as 30 directions have also been reported. While 6 directions are the bare minimum required for tensor calculation, other supplemental criteria have been suggested; for example, a minimum of 12 directions have been suggested to eliminate orientation bias in tensor results [81]. As a range of optimization studies have investigated, parameter estimation quality (both accuracy and precision) depends crucially on sufficient signal-to-noise ratio (SNR) [16, 17, 82, 83]. While thresholds and criteria vary, minimum SNR levels of 20-30 are frequently suggested for advanced renal DWI.

Table 1 shows the results of the diffusion biomarker vs. protocol parameter correlations in healthy volunteers. All correlation results are shown for individual protocol parameters and biomarkers, and additional correlations are shown with protocol parameter combinations that provided higher correlation coefficients. The primary protocol element correlation with reported diffusion biomarkers is average $b$ value, which significantly correlated negatively with tissue diffusivity $D[\mathrm{Cx}$ (cortex): $R=-0.506$, $p=0.03$; Md (medulla): $R=-0.528, p=0.02]$ and positively with flow fraction $f(\mathrm{Cx}: R=0.687, p=0.002$; Md: $R=0.566, p=0.01)$. These correlations may have contributions from partial sampling of the IVIM signal response, with higher $b$ value ranges providing better estimates of both slow and fast diffusion components. Conversely, if $b$ values are sampled beyond the appropriate SNR level, Rician noise [84] (or more complex noise patterns accompanying image reconstruction [85]) bias can lower ADC or $D$ values and inflate $f$ values. Similar negative correlation trends $(p<0.1)$ are seen between cortical $\operatorname{ADC}(R=-0.378, p=0.08)$ or cortical MD $(R=-0.531$, $p=0.05)$ and maximum $\mathrm{b}$ value. Transverse relaxation effects cause secondary correlations of flowing fraction with echo time TE $(R=0.474, p=0.055)$ or equivalently T2 decay factor $(R=-0.495, p=0.04)$, likely due to reduction of the more rapidly relaxing tissue compartment, as quantified by Lemke et al. [17], and supported by the disparate relaxation times of renal tissue [79] and serum blood [86] or urine [87-89]. Another potential modulator of contrast is diffusion time, which is lengthened at larger echo time, though the role of this parameter in renal tissue has not been conclusively mapped out. Combining $b$ value and sequence timing factors together showed some amplified correlations, particularly for flow fraction and tissue diffusivity. In some cases, increasing $\mathrm{T} 1$ recovery increased flow representation and therefore higher $f$ and ADC. Finally, a combination of relaxation factors and average $b$ value showed a negative correlation trend $(R=-0.463, p=0.07)$ with medullary FA, consistent with a modulation in flow effects on diffusion anisotropy. Figure 2 shows example correlations between renal DWI biomarkers in the literature and protocol parameters. As these variations of acquisition protocols and DWI biomarkers should be avoided in the translation of renal DWI to clinical practice, the present manuscript describes ongoing efforts to maximize lessons learned from existing work to facilitate multi-site consistency through standardized acquisition, analysis, and reporting guidelines.

Table 2 shows summarized diffusion biomarkers in cortex and medulla in healthy volunteers from the literature review, stratified by field strength $(1.5 \mathrm{~T}$ or $3.0 \mathrm{~T})$. The only cases showing significant differences were IVIM pseudodiffusion $\left(D^{*}\right)$ and DTI mean diffusivity (MD) in cortex, both of which were higher at $1.5 \mathrm{~T}$ than $3.0 \mathrm{~T}$.

\section{Survey results}

The second-round survey included 21 respondents from 21 institutions in 8 different countries on 3 continents. 9 of 21 (43\%) were radiologists, while 13/21 (57\%) were physicists (11), biomedical engineers (1), or mathematicians (1). $71 \%$ of the respondents used renal diffusion for volunteer research, $76 \%$ used it for patient research, 38\% used it for clinical practice, and $14 \%$ used it for clinical trials.

For the second-round survey, among the 87 questions testing levels of agreement, 23 reached consensus agreement and 18 reached consensus disagreement. These results also resolved 16 other questions on the same topics as the "parent" consensus questions. For the remaining questions, if preferences are made for 17 questions, the remaining 13 questions are resolved. The fully aggregated survey responses, as well as text comments provided, are included as supplementary material, with Table 3 summarizing results of agree/disagree questions (with those reaching consensus 
Table 1 Correlations between reported renal diffusion metrics in the literature from cortex $(\mathrm{Cx})$ or medulla $(\mathrm{Md})$ regions of healthy volunteer kidneys and the corresponding studies' protocol parameters average $b$ value (ave $b$ val), maximum $b$ value (max $b$ val), echo time
(TE), repetition time (TR), T2-weighting factor (T2f), and T1-weighting factor (T1f) (see text for calculation of relaxation weighting factors)

\begin{tabular}{|c|c|c|c|c|c|c|c|c|c|c|c|c|c|c|c|c|c|c|}
\hline & \multicolumn{3}{|l|}{$\mathrm{ADC}$} & \multicolumn{3}{|l|}{$D$} & \multicolumn{3}{|l|}{$f$} & \multicolumn{3}{|l|}{$D^{*}$} & \multicolumn{3}{|l|}{ MD } & \multicolumn{3}{|l|}{ FA } \\
\hline & $R$ & $p$ & $N$ & $R$ & $p$ & $N$ & $R$ & $p$ & $N$ & $R$ & $p$ & $N$ & $R$ & $p$ & $N$ & $R$ & $p$ & $N$ \\
\hline \multicolumn{19}{|c|}{ Ave $b$ val } \\
\hline $\mathrm{Cx}$ & -0.162 & 0.47 & 22 & -0.506 & 0.03 & 18 & 0.687 & 0.002 & 18 & -0.268 & 0.40 & 12 & -0.147 & 0.62 & 14 & 0.144 & 0.60 & 16 \\
\hline Md & -0.154 & 0.56 & 17 & -0.528 & 0.02 & 18 & 0.566 & 0.01 & 18 & -0.319 & 0.31 & 12 & 0.093 & 0.75 & 14 & -0.296 & 0.27 & 16 \\
\hline \multicolumn{19}{|c|}{ Max $b$ val } \\
\hline $\mathrm{Cx}$ & -0.378 & 0.08 & 22 & -0.245 & 0.33 & 18 & 0.285 & 0.25 & 18 & 0.106 & 0.74 & 12 & -0.531 & 0.05 & 14 & 0.102 & 0.71 & 16 \\
\hline Md & -0.223 & 0.39 & 17 & -0.260 & 0.30 & 18 & 0.281 & 0.26 & 18 & 0.239 & 0.46 & 12 & 0.161 & 0.58 & 14 & -0.192 & 0.45 & 16 \\
\hline \multicolumn{19}{|l|}{ TE } \\
\hline $\mathrm{Cx}$ & 0.220 & 0.37 & 19 & 0.149 & 0.57 & 17 & 0.474 & 0.055 & 17 & -0.262 & 0.44 & 11 & 0.036 & 0.90 & 14 & -0.087 & 0.75 & 16 \\
\hline Md & 0.345 & 0.19 & 16 & 0.163 & 0.53 & 17 & 0.150 & 0.57 & 17 & -0.272 & 0.42 & 11 & -0.152 & 0.61 & 14 & 0.216 & 0.42 & 16 \\
\hline \multicolumn{19}{|l|}{$\mathrm{TR}$} \\
\hline $\mathrm{Cx}$ & 0.225 & 0.44 & 14 & -0.270 & 0.30 & 17 & 0.097 & 0.71 & 17 & 0.392 & 0.23 & 11 & -0.168 & 0.57 & 14 & 0.038 & 0.89 & 16 \\
\hline $\mathrm{Md}$ & 0.039 & 0.90 & 12 & -0.186 & 0.48 & 17 & 0.043 & 0.87 & 17 & 0.459 & 0.16 & 11 & -0.060 & 0.84 & 14 & -0.292 & 0.27 & 16 \\
\hline \multicolumn{19}{|l|}{$T 2 f$} \\
\hline $\mathrm{Cx}$ & -0.223 & 0.36 & 19 & -0.151 & 0.56 & 17 & -0.495 & 0.04 & 17 & 0.249 & 0.46 & 11 & -0.083 & 0.78 & 14 & 0.102 & 0.71 & 16 \\
\hline $\mathrm{Md}$ & -0.378 & 0.15 & 16 & -0.158 & 0.55 & 17 & -0.155 & 0.55 & 17 & 0.257 & 0.45 & 11 & 0.127 & 0.67 & 14 & -0.255 & 0.34 & 16 \\
\hline \multicolumn{19}{|l|}{ T1f } \\
\hline $\mathrm{Cx}$ & -0.016 & 0.96 & 14 & -0.142 & 0.59 & 17 & 0.171 & 0.51 & 17 & 0.298 & 0.37 & 11 & -0.268 & 0.35 & 14 & 0.126 & 0.64 & 16 \\
\hline Md & 0.110 & 0.73 & 12 & -0.129 & 0.62 & 17 & 0.018 & 0.95 & 17 & 0.274 & 0.42 & 11 & -0.116 & 0.69 & 14 & 0.079 & 0.77 & 16 \\
\hline \multicolumn{19}{|c|}{ Ave $b \times \mathrm{TE}$} \\
\hline $\mathrm{Cx}$ & 0.007 & 0.98 & 19 & -0.338 & 0.19 & 17 & 0.713 & 0.001 & 17 & -0.395 & 0.23 & 11 & -0.168 & 0.57 & 14 & 0.086 & 0.75 & 16 \\
\hline Md & 0.506 & 0.046 & 16 & -0.331 & 0.20 & 17 & 0.501 & 0.04 & 17 & -0.457 & 0.16 & 11 & 0.001 & 1.0 & 14 & -0.216 & 0.42 & 16 \\
\hline \multicolumn{19}{|c|}{ Ave $b \times \mathrm{T} 2 \mathrm{f}$} \\
\hline $\mathrm{Cx}$ & -0.067 & 0.78 & 19 & -0.701 & 0.003 & 17 & 0.435 & 0.08 & 17 & -0.184 & 0.59 & 11 & -0.106 & 0.72 & 14 & 0.200 & 0.46 & 16 \\
\hline Md & 0.107 & 0.69 & 16 & -0.741 & 0.001 & 17 & 0.559 & 0.02 & 17 & -0.228 & 0.50 & 11 & 0.167 & 0.57 & 14 & -0.357 & 0.18 & 16 \\
\hline \multicolumn{19}{|c|}{ Ave $b \times \mathrm{T} 2 \mathrm{f} / \mathrm{T} 1 \mathrm{f}$} \\
\hline $\mathrm{Cx}$ & 0.262 & 0.37 & 14 & -0.479 & 0.05 & 17 & 0.223 & 0.39 & 17 & -0.338 & 0.31 & 11 & 0.141 & 0.63 & 14 & 0.030 & 0.91 & 16 \\
\hline $\mathrm{Md}$ & 0.175 & 0.59 & 12 & -0.503 & 0.04 & 17 & 0.427 & 0.09 & 17 & -0.340 & 0.31 & 11 & 0.253 & 0.38 & 14 & -0.463 & 0.07 & 16 \\
\hline \multicolumn{19}{|c|}{ Ave $b \times \mathrm{T} 1 \mathrm{f} / \mathrm{T} 2 \mathrm{f}$} \\
\hline $\mathrm{Cx}$ & 0.691 & 0.006 & 14 & -0.381 & 0.13 & 17 & 0.682 & 0.003 & 17 & -0.174 & 0.61 & 11 & -0.269 & 0.35 & 14 & 0.133 & 0.63 & 16 \\
\hline Md & 0.660 & 0.02 & 12 & -0.369 & 0.15 & 17 & 0.477 & 0.05 & 17 & -0.240 & 0.48 & 11 & -0.049 & 0.87 & 14 & -0.204 & 0.45 & 16 \\
\hline \multicolumn{19}{|c|}{ Max $b \times \mathrm{T} 1 \mathrm{f} / \mathrm{T} 2 \mathrm{f}$} \\
\hline $\mathrm{Cx}$ & 0.110 & 0.71 & 14 & -0.155 & 0.55 & 17 & 0.547 & 0.02 & 17 & 0.171 & 0.62 & 11 & -0.539 & 0.047 & 14 & 0.088 & 0.75 & 16 \\
\hline Md & 0.293 & 0.36 & 12 & -0.150 & 0.57 & 17 & 0.310 & 0.23 & 17 & 0.236 & 0.48 & 11 & -0.015 & 0.96 & 14 & -0.163 & 0.55 & 16 \\
\hline
\end{tabular}

Pearson correlation coefficients $R$, significance levels from two-sided $t$ test, $p$, and number of studies contributing $N$ are shown for the following diffusion parameters: apparent diffusion coefficient (ADC), IVIM tissue diffusivity $(D)$, IVIM flow fraction $(f)$, IVIM pseudodiffusivity $\left(D^{*}\right)$, DTI mean diffusivity (MD), and DTI fractional anisotropy (FA). Significant correlations $(p<0.05)$ are highlighted in bold and moderate trends $(p<0.1)$ in italics

highlighted). Regarding magnetic field strength, a consensus majority $(81 \%)$ responded either $1.5 \mathrm{~T}$ or $3.0 \mathrm{~T}$ as acceptable. Regarding reporting preferences, all suggested acquisition details (matrix, image orientation, fat suppression mode, averages, slice thickness, resolution, field of view, TR, TE, number and choice of $b$ values, and number of directions) received consensus support to be reported. Reporting biomarkers in both cortex and medulla was supported by consensus. Regarding processing, motion correction algorithm, processing software, IVIM fit algorithm, and IVIM fit option received consensus support to be reported. Regarding biomarkers' summary statistics, mean, median, and standard deviation values received consensus support to be reported. There are a range of topics that did not reach the level of consensus, including slice thickness, repetition time TR, number of signal averages, breathing mode, separate 

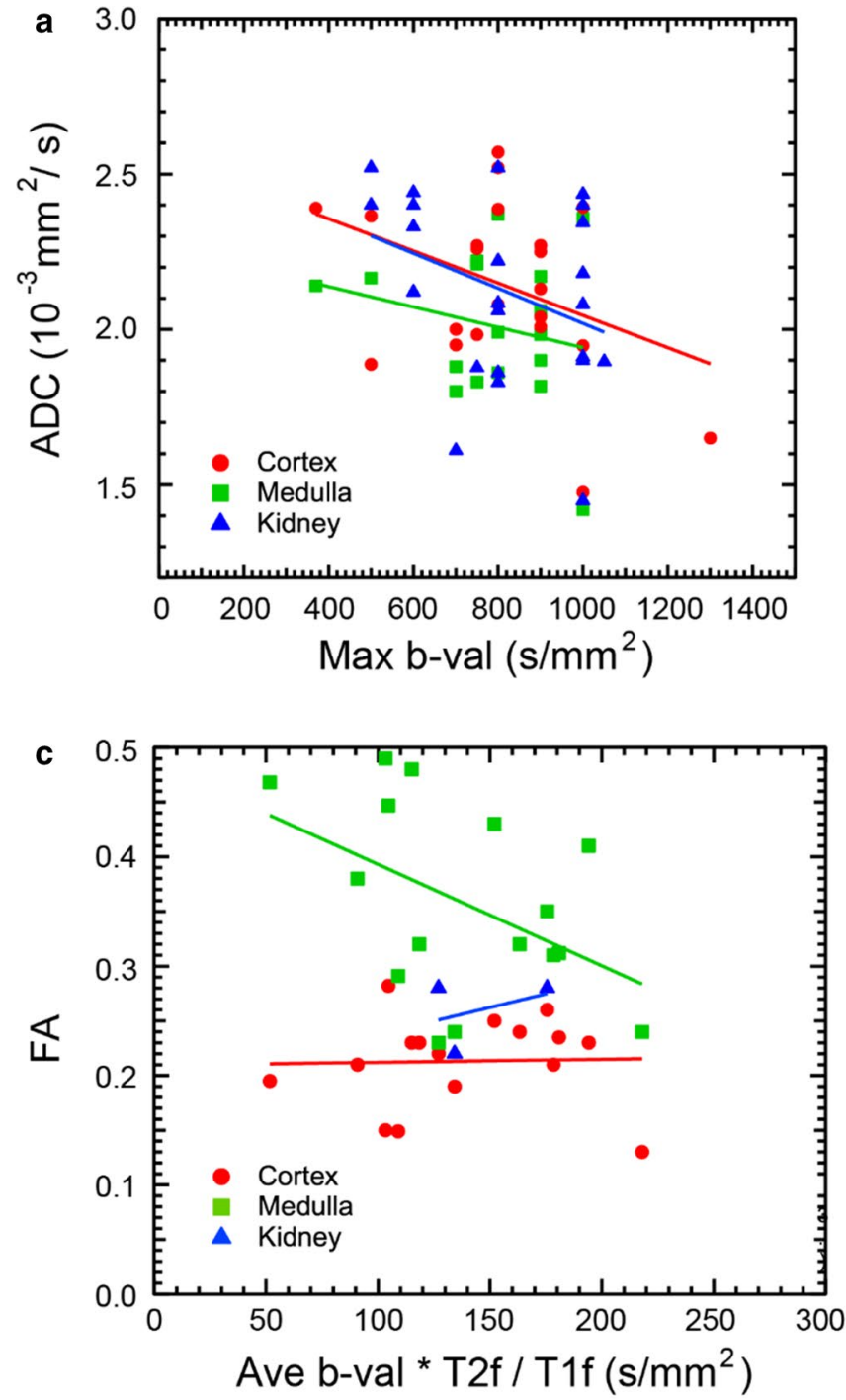

Fig. 2 Correlations between renal diffusion MRI metrics and protocol parameters from the literature from cortex, medulla, and whole kidney tissue in healthy adults. a ADC, b IVIM tissue diffusivity $D$, c DTI fractional anisotropy FA, and d IVIM flow fraction $f$ vs. aver-

vs. combined protocols, diffusion gradient waveform, the number and highest $b$ value employed, number of diffusion directions for DTI, and aspects of ROI prescriptions.

Considering the literature trends, consensus views, preferences, comments and practical aspects surrounding future evidence generation, recommendations are given in Table 4 for monoexponential DWI, IVIM, and DTI protocols. For many of the issues guiding protocol selection, the survey process provided clear indications of consensus choices (Table 3). For those topics not reaching consensus, we combine lesser-weighted preferences, practical issues, and information from text survey responses to synthesize recommendations. For acquisition, the consensus includes pulse sequences, RF coils, in-plane matrix/resolution, slice
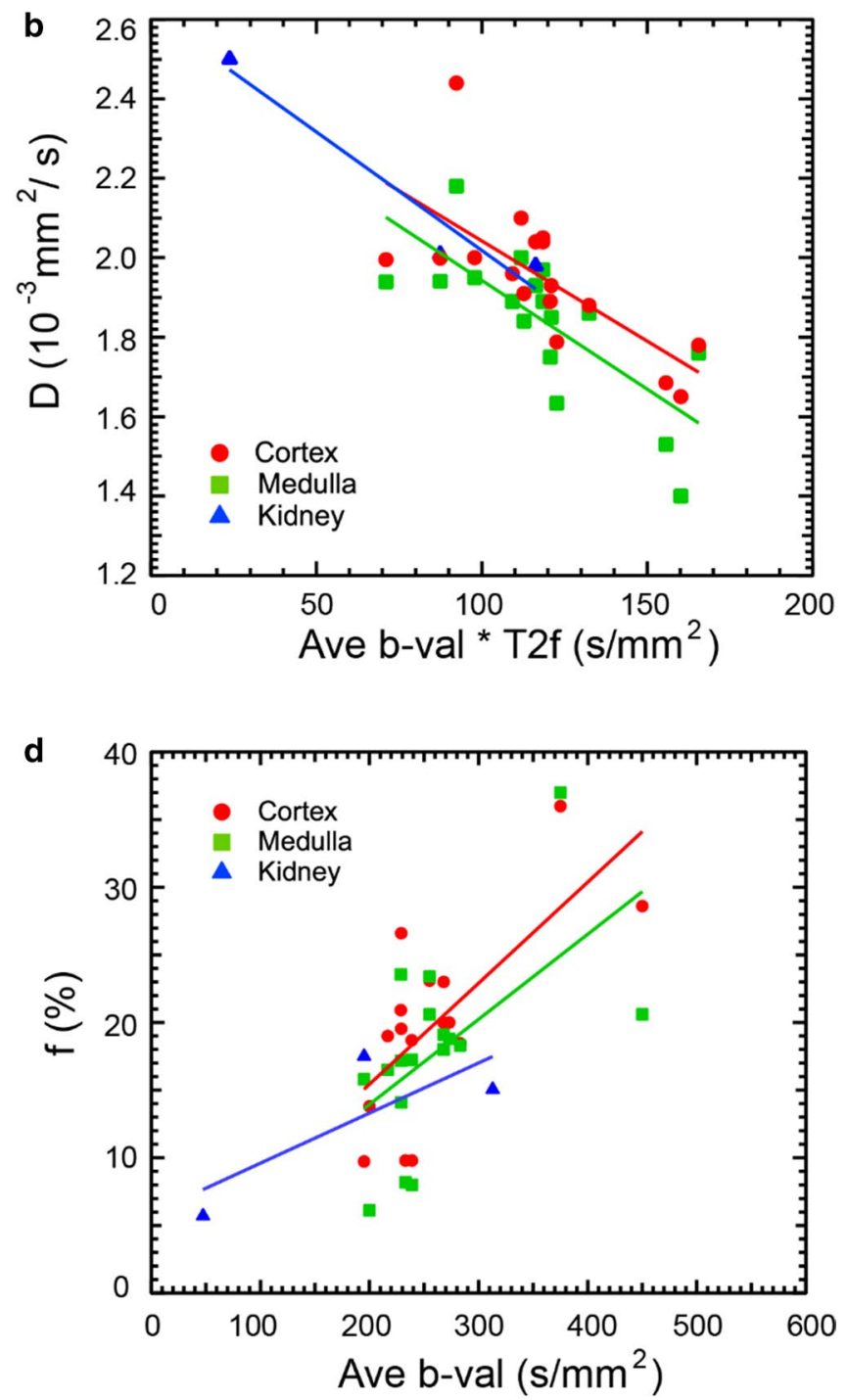

age or maximum $b$ value with relaxation weighting terms. Inter-study variation can be reduced when desired for larger evidence generation using more standardized protocols

coverage, parallel imaging acceleration, fat suppression, echo time, and absence of cardiac gating. Strong preference (62\%) was given for $>4 \mathrm{~mm}$ slice thickness, though some respondents expressed a desire for lower values when feasible. Strong preference (67\% agreement) was also given for a $\mathrm{TR}=2-4 \mathrm{~s}$. Given some contribution of T1 weighting to parameter variability, we have suggested a standardized repetition time $\mathrm{TR}=4 \mathrm{~s}$. Breathing mode did not reach consensus; however, strong preference $(70 \%)$ was given to respiratory gating and free breathing $(66 \%)$. Free breathing was noted to be acceptable in cases of renal allograft imaging. We have recommended respiratory gating when available and free breathing with post hoc unilateral motion correction when not available (which was separately recommended by 
Table 2 Comparisons between reported renal diffusion metrics in the literature from cortex or medulla regions of healthy volunteer kidneys at different field strengths $(1.5$ or $3.0 \mathrm{~T})$

\begin{tabular}{lllllll}
\hline & ADC & $D$ & $f$ & $D^{*}$ & MD & FA \\
\hline Cortex & & & & & \\
$1.5 \mathrm{~T}$ & & & & & \\
$\quad$ Mean \pm SD & $2056 \pm 285$ & $1966 \pm 72$ & $19.9 \pm 3.2$ & $50,800 \pm 13,454$ & $2508 \pm 86$ & $0.208 \pm 0.045$ \\
$N$ & 12 & 7 & 7 & 4 & 4 & 4 \\
$3.0 \mathrm{~T}$ & & & & & & \\
Mean $\pm \mathrm{SD}$ & $2243 \pm 225$ & $1919 \pm 229$ & $20.1 \pm 8.4$ & $24,964 \pm 20,298$ & $2262 \pm 164$ & $0.215 \pm 0.043$ \\
$N$ & 10 & 11 & 11 & 8 & 10 & 12 \\
$p$ & 0.100 & 0.538 & 0.944 & $\mathbf{0 . 0 2 8}$ & $\mathbf{0 . 0 0 4}$ & 0.779 \\
Medulla & & & & & & \\
$1.5 \mathrm{~T}$ & & & & & & \\
Mean $\pm \mathrm{SD}$ & $1987 \pm 267$ & $1884 \pm 76$ & $17.5 \pm 5.5$ & $57,350 \pm 25,505$ & $2348 \pm 589$ & $0.425 \pm 0.079$ \\
$N$ & 8 & 7 & 7 & 4 & 4 & 4 \\
$3.0 \mathrm{~T}$ & & & & & & \\
Mean $\pm \mathrm{SD}$ & $2031 \pm 227$ & $1796 \pm 228$ & $18.0 \pm 7.8$ & $29,016 \pm 19,272$ & $2092 \pm 162$ & $0.335 \pm 0.082$ \\
$N$ & 9 & 11 & 11 & 8 & 10 & 12 \\
$p$ & 0.721 & 0.261 & 0.877 & 0.110 & 0.452 & 0.105 \\
\hline
\end{tabular}

Mean and standard deviation values, significance levels from two-sided $t$ test, $p$, and number of studies contributing $N$ are shown for the following diffusion parameters: apparent diffusion coefficient (ADC), IVIM tissue diffusivity $(D)$, IVIM flow fraction $(f)$, IVIM pseudodiffusivity $\left(D^{*}\right)$, DTI mean diffusivity (MD), and DTI fractional anisotropy (FA). Significant field differences $(p<0.05)$ are highlighted in bold. ADC, $D, D^{*}$, and MD values are given in $10^{-6} \mathrm{~mm}^{2} / \mathrm{s}, f$ is given in $\%$, and FA is unitless consensus). Regarding field strength, consensus approval for either $1.5 \mathrm{~T}$ or $3.0 \mathrm{~T}$ was found $(81 \%)$, and only minimal differences were observed in the literature (Table 2). The SNR advantage of higher field is balanced by other disadvantages for DWI such as susceptibility-induced image distortion. Correspondingly, either field strength is deemed acceptable and investigators suggested to employ whichever is better equipped with hardware or software elements consistent with recommendations herein.

\section{Discussion}

The design of diffusion MRI protocols for renal imaging remains controversial, with some support for separate protocols for each diffusion technique and slightly more support for combined protocols. Similarly, separate protocols for 'standardized' efforts and exploratory research had only $50 \%$ support. Since deriving all measures from a combined protocol requires more sophisticated workflows than are universally available and consistent with the goals of generating generalizable evidence, we have thus recommended parsimonious protocols for monoexponential DWI, IVIM, and DTI studies. As noted below, however, the encoding parameters suggested have commonalities (e.g., $b$ values) that may allow pooling of analogous biomarkers and consistency with advanced protocols involving combined encoding.

As mentioned in "Results", field strength was not a crucial determining factor in either the diffusion metrics reported in the literature (only 2 out of 24 comparisons showed significant differences in Table 2) or in the consensus preferences of the survey respondents. The field differences observed in $D^{*}$ and MD in cortex in the literature values most likely arise from indirect effects of differential relaxation weighting of flow and structural compartments with field, since both have field-dependent relaxation times as discussed above. Thus, currently field strength is not a stringent requirement for standardization, although the growing technological prevalence of higher field $(3.0 \mathrm{~T})$ may make the point moot.

Diffusion weighting (choice of $b$ values) is a crucial element of diffusion MRI protocols. For monoexponential DWI studies, consensus was found for more than $2 b$ values, including values $<200 \mathrm{~s} / \mathrm{mm}^{2}$, with strong preference for a maximum $b$ value of $800 \mathrm{~s} / \mathrm{mm}^{2}$. For IVIM studies, consensus was found for a number of $b$ values greater than $6 b$ values, with highest preference for more than 8 . Finally, for DTI studies, preference was given to more than $2 b$ values $(61 \%)$, with a maximum $b$ value of $600 \mathrm{~s} / \mathrm{mm}^{2}(59 \%)$. Six directions were deemed insufficient for DTI (76\%), with a slight preference for more than 12 directions (63\%). In addition to these indications from our panel, we may also take guidance from optimization studies on renal DWI sampling $[16,17]$ that emphasized the importance of several key $b$ value regimes: low $\left(0-200 \mathrm{~s} / \mathrm{mm}^{2}\right)$ intermediate $(200-400 \mathrm{~s} /$ $\mathrm{mm}^{2}$ ), and high $\left(600-800 \mathrm{~s} / \mathrm{mm}^{2}\right)$. Finally, we deem it valuable to suggest common encoding parameters between techniques (monoexponential DWI, IVIM, DTI) where possible 
Table 3 Summary of survey results on agree/disagree questions

\begin{tabular}{|c|c|c|c|c|c|c|}
\hline \multirow[b]{2}{*}{ Question } & \multicolumn{3}{|c|}{ All responses } & \multicolumn{2}{|c|}{ W/o abstentions } & \multirow[t]{2}{*}{ Choice } \\
\hline & $\%$ Agree & $\begin{array}{c}\% \\
\text { No basis }\end{array}$ & $\begin{array}{c}\% \\
\text { Disagree }\end{array}$ & $\%$ Agree & $\%$ Disagree & \\
\hline Diet needs to be controlled before the scan & 33.3 & 28.6 & 38.1 & 46.7 & 53.3 & \\
\hline $\begin{array}{l}\text { Subject should be scanned in a normal hydration } \\
\text { status when clinically appropriate }\end{array}$ & 76.2 & 19.1 & 4.8 & 94.1 & 5.9 & Agree \\
\hline $\begin{array}{l}\text { Subjects are required to follow a controlled and } \\
\text { standardized salt intake before the scan }\end{array}$ & 4.8 & 42.9 & 52.4 & 8.3 & 91.7 & Disagree \\
\hline Single-shot echo planar imaging sequence & 100 & 0 & 0 & 100 & 0 & Agree \\
\hline Multi-shot echo planar imaging sequence & 28.6 & 33.3 & 38.1 & 42.9 & 57.1 & \\
\hline RF body matrix coils & 95.2 & 4.8 & 0 & 100 & 0 & Agree \\
\hline Axial slice orientation & 28.6 & 4.8 & 66.7 & 30 & 70 & Disagree \\
\hline Coronal slice orientation (consistent with above) & 42.9 & 9.5 & 47.6 & 47.4 & 52.6 & \\
\hline $\begin{array}{l}\text { Oblique coronal slice orientation along long kidney } \\
\text { axis (consistent with above) }\end{array}$ & 76.2 & 0 & 23.8 & 76.2 & 23.8 & Agree \\
\hline Acquired matrix size $>128$ & 85.7 & 0 & 14.3 & 85.7 & 14.3 & Agree \\
\hline Inplane resolution $2 \mathrm{~mm}$ or smaller & 19.1 & 0 & 81.0 & 19.1 & 81.0 & Disagree \\
\hline Inplane resolution between 2 and $3 \mathrm{~mm}$ & 90.5 & 0 & 9.5 & 90.5 & 9.5 & Agree \\
\hline Inplane resolution $>\mathbf{3} \mathrm{mm}$ & 4.8 & 9.5 & 85.7 & 5.3 & 94.7 & Disagree \\
\hline Slice thickness $2 \mathrm{~mm}$ or less & 0 & 9.5 & 90.5 & 0 & 100 & Disagree \\
\hline Slice thickness between 2 and $4 \mathrm{~mm}$ & 57.1 & 4.8 & 38.1 & 60 & 40 & Agree \\
\hline Slice thickness $>4 \mathrm{~mm}$ & 61.9 & 0 & 38.1 & 61.9 & 38.1 & Agree \\
\hline Gap between slices & 42.9 & 4.8 & 52.4 & 45 & 55 & \\
\hline Full kidney slice coverage & 90.5 & 0 & 9.5 & 90.5 & 9.5 & Agree \\
\hline Parallel imaging acceleration (factor 2 ) & 95.2 & 0 & 4.8 & 95.2 & 4.8 & Agree \\
\hline $\begin{array}{l}\text { Parallel imaging acceleration (factor }>2 \text { ) (consistent } \\
\text { with above) }\end{array}$ & 19.1 & 23.81 & 57.1 & 25 & 75 & Disagree \\
\hline SPAIR fat suppression & 61.9 & 23.8 & 14.3 & 81.3 & 18.8 & Agree \\
\hline STIR fat suppression (consistent with above) & 9.5 & 28.6 & 61.9 & 13.3 & 86.7 & Disagree \\
\hline $2000 \mathrm{~ms}<\mathrm{TR}<4000 \mathrm{~ms}$ & 66.7 & 0 & 33.3 & 66.7 & 33.3 & Agree \\
\hline $\mathrm{TR}>4000 \mathrm{~ms}$ & 38.1 & 4.8 & 57.1 & 40 & 60 & Disagree \\
\hline $\mathrm{TE}<100 \mathrm{~ms}$ & 95.2 & 0 & 4.8 & 95.2 & 4.8 & Agree \\
\hline TE minimum allowed by hardware / sequence & 85.7 & 9.5 & 4.8 & 94.7 & 5.3 & Agree \\
\hline 2 signal averages & 38.1 & 4.8 & 57.1 & 40 & 60 & Disagree \\
\hline 3 signal averages & 66.7 & 0 & 33.3 & 66.7 & 33.3 & Agree \\
\hline
\end{tabular}


Table 3 (continued)

\begin{tabular}{|c|c|c|c|c|c|c|}
\hline Expiration Breathhold acquisition & 9.5 & 4.8 & 85.7 & 10 & 90 & Disagree \\
\hline Free breathing acquisition (consistent with above) & 66.7 & 0 & 33.3 & 66.7 & 33.3 & Agree \\
\hline Respiratory gated acquisition (consistent with above) & 66.7 & 4.8 & 28.6 & 70 & 30 & Agree \\
\hline Cardiac gating (systole) & 0 & 33.3 & 66.7 & 0 & 100 & Disagree \\
\hline Cardiac gating (diastole) & 4.8 & 33.3 & 61.9 & 7.1 & 92.9 & Disagree \\
\hline $\begin{array}{l}\text { Separate acquisitions for ADC / IVIM vs. DTI studies } \\
\text { in a multiparametric protocol }\end{array}$ & 42.9 & 9.5 & 47.6 & 47.4 & 52.6 & \\
\hline $\begin{array}{l}\text { Separate protocols for multiparametric acquisitions and } \\
\text { exploratory renal diffusion MRI research }\end{array}$ & 47.6 & 4.8 & 47.6 & 50 & 50 & \\
\hline $\begin{array}{l}\text { Single protocol to provide all metrics (ADC, DTI, } \\
\text { IVIM) }\end{array}$ & 47.6 & 14.3 & 38.1 & 55.6 & 44.4 & \\
\hline Monopolar diffusion gradients & 57.1 & 19.1 & 23.8 & 70.6 & 29.41 & Agere \\
\hline Twice-refocused Bipolar diffusion gradients & 28.6 & 42.9 & 28.6 & 50 & 50 & \\
\hline DWI sequence with only 2 b-values & 14.3 & 0 & 85.7 & 14.3 & 85.7 & Disagree \\
\hline DWI sequence with more than 2 b-values & 90.5 & 0 & 9.5 & 90.5 & 9.5 & Agree \\
\hline ADC studies : include low b-values $<200 \mathrm{~s} / \mathrm{mm} 2$ & $\mathbf{8 5 . 7}$ & 0 & 14.3 & 85.7 & 14.3 & Agree \\
\hline ADC studies : highest b-value $600 \mathrm{~s} / \mathrm{mm} 2$ & 19.1 & 0 & 81.0 & 19.1 & 81.0 & Disagree \\
\hline $\begin{array}{l}\text { ADC studies : highest b-value } 800 \mathrm{~s} / \mathrm{mm} 2 \text { (consistent } \\
\text { with above) }\end{array}$ & 61.9 & 0 & 38.1 & 61.9 & 38.1 & Agree \\
\hline $\begin{array}{l}\text { ADC studies : high b-value } 1000 \mathrm{~s} / \mathrm{mm} 2 \text { (consistent } \\
\text { with above) }\end{array}$ & 28.6 & 4.8 & 66.7 & 30 & 70 & Disagree \\
\hline IVIM studies : 4 b-values & 0 & 9.5 & 90.5 & 0 & 100 & Disagree \\
\hline IVIM studies : 6 b-values & 19.1 & 9.5 & 71.4 & 21.1 & 78.9 & Disagree \\
\hline IVIM studies : $8 \mathrm{~b}$-values & 47.6 & 9.5 & 42.9 & 52.6 & 47.4 & \\
\hline IVIM studies : $>8$ b-values & 52.4 & 4.8 & 42.9 & 55 & 45 & \\
\hline DTI studies : 2 b-values & 38.1 & 28.6 & 33.3 & 53.3 & 46.7 & \\
\hline DTI studies: $>2$ b-values & 38.1 & 38.1 & 23.81 & 61.51 & 38.5 & Agree \\
\hline DTI studies : highest b-value $400 \mathrm{~s} / \mathrm{mm} 2$ & 4.8 & 28.6 & 66.7 & 6.7 & 93.3 & Disagree \\
\hline DTI studies : highest b-value $600 \mathrm{~s} / \mathrm{mm} 2$ & 47.6 & 19.1 & 33.3 & 58.8 & 41.2 & \\
\hline DTI studies : highest b-value $800 \mathrm{~s} / \mathrm{mm} 2$ & 38.1 & 23.8 & 38.1 & 50 & 50 & \\
\hline DTI studies : highest b-value $1000 \mathrm{~s} / \mathrm{mm} 2$ & 9.5 & 28.6 & 61.9 & 13.3 & 86.7 & Disagree \\
\hline DTI studies : 6 directions & 19.1 & 19.1 & 61.91 & 23.51 & 76.5 & Disagree \\
\hline DTI studies : 12 directions & 42.9 & 28.6 & 28.6 & 60 & 40 & Agree \\
\hline
\end{tabular}


Table 3 (continued)

\begin{tabular}{|c|c|c|c|c|c|c|}
\hline DTI studies : $>12$ directions & 47.6 & 23.8 & 28.6 & 62.5 & 37.5 & Agree \\
\hline Post-hoc EPI distortion correction & 52.4 & 38.1 & 9.5 & 84.6 & 15.4 & Agree \\
\hline Post-hoc motion correction / registration & 90.5 & 9.5 & 0 & 100 & 0 & Agree \\
\hline Unilateral motion correction / registration & 47.6 & 38.1 & 14.3 & 76.9 & 23.1 & Agree \\
\hline Manual ROI placement & 85.7 & 9.5 & 4.8 & 94.7 & 5.3 & Agree \\
\hline Manual ROI placement on ADC map & 42.9 & 9.5 & 47.6 & 47.4 & 52.6 & \\
\hline Manual ROI placement on b0 & 76.2 & 9.5 & 14.3 & 84.2 & 15.8 & Agree \\
\hline Manual ROI placement on FA map & 42.9 & 14.3 & 42.9 & 50 & 50 & \\
\hline Manual continuous cortical stripe ROI per slice & 47.6 & 19.1 & 33.3 & 58.8 & 41.2 & \\
\hline Manual Whole medulla ROI per slice & 23.8 & 19.1 & 57.1 & 29.4 & 70.6 & Disagree \\
\hline Manual Whole kidney ROI per slice & 33.3 & 14.3 & 52.4 & 38.9 & 61.1 & Disagree \\
\hline Manual Multiple medulla ROI per slice & 76.2 & 9.5 & 14.3 & 84.2 & 15.8 & Agree \\
\hline Manual Multiple cortical ROIs per slice & 52.4 & 9.5 & 38.1 & 57.9 & 42.1 & \\
\hline If multiple, Three cortical ROIs per slice & 38.1 & 19.1 & 42.9 & 47.1 & 52.9 & \\
\hline If multiple, Three medulla ROIs per slice & 47.6 & 19.1 & 33.3 & 58.8 & 41.2 & \\
\hline If multiple, $>$ Three cortical ROIs per slice & 28.6 & 14.3 & 57.1 & 33.3 & 66.7 & Disagree \\
\hline If multiple, $>$ Three medullary ROIs per slice & 33.3 & 14.3 & 52.4 & 38.9 & 61.1 & Disagree \\
\hline If multiple slices, 3 slices sampled & 52.4 & 9.5 & 38.1 & 57.9 & 42.1 & \\
\hline If multiple slices, $>3$ slices sampled & 57.1 & 9.5 & 33.3 & 63.2 & 36.8 & Agree \\
\hline Automatic ROI placement, based on b0 histogram & 23.8 & 38.1 & 38.1 & 38.5 & 61.5 & Disagree \\
\hline $\begin{array}{l}\text { Automatic ROI placement, based on ADC } \\
\text { histogram }\end{array}$ & 14.3 & 42.9 & 42.9 & 25 & 75 & Disagree \\
\hline Automatic ROI placement, based on FA histogram & 19.1 & 42.9 & 38.1 & 33.3 & 66.7 & Disagree \\
\hline Report diffusion biomarkers in cortex & 95.2 & 4.8 & 0 & 100 & 0 & Agree \\
\hline Report diffusion biomarkers in medulla & 90.5 & 9.5 & 0 & 100 & 0 & Agree \\
\hline Report diffusion biomarkers in whole kidney & 38.1 & 14.3 & 47.6 & 44.4 & 55.6 & \\
\hline Diffusion units $10^{\wedge}-3 \mathrm{~mm} 2 / \mathrm{s}$ & 81.0 & 0 & 19.1 & 81.0 & 19.1 & Agree \\
\hline Diffusion units $10^{\wedge}-6 \mathrm{~mm} 2 / \mathrm{s}$ & 28.6 & 0 & 71.4 & 28.6 & 71.4 & Disagree \\
\hline Diffusion units microns ${ }^{\wedge} 2 / \mathrm{ms}$ & 14.3 & 0 & 85.7 & 14.3 & 85.7 & Disagree \\
\hline Colormap presentation & 76.2 & 0 & 23.8 & 76.2 & 23.8 & Agree \\
\hline Grayscale map presentation & 61.9 & 0 & 38.1 & 61.9 & 38.1 & Agree \\
\hline Parametric map fusion with anatomic imaging & 61.9 & 9.5 & 28.6 & 68.4 & 31.6 & Agree \\
\hline
\end{tabular}

Questions highlighted in bold achieved consensus $(\geq 75 \%)$. The choice on each question (agree or disagree) is labeled and color coded; green $=$ consensus $(\geq 75 \%)$; orange $=$ preference $(\geq 60 \%)$; red $=$ indeterminate 
Table 4 Recommendations for acquisition and processing of renal DWI data

\begin{tabular}{|c|c|c|c|c|}
\hline Protocol option & \multicolumn{3}{|c|}{ Recommendation } & Weight \\
\hline Preparation & \multicolumn{3}{|c|}{ Normal hydration } & \\
\hline Field strength & \multicolumn{3}{|c|}{$1.5 \mathrm{~T}$ or $3.0 \mathrm{~T}$} & \\
\hline Sequence & \multicolumn{3}{|c|}{ Single shot EPI } & \\
\hline Orientation & \multicolumn{3}{|c|}{ Oblique coronal } & \\
\hline Matrix & \multicolumn{3}{|c|}{$>128$} & \\
\hline In-plane resolution & \multicolumn{3}{|c|}{$2-3 \mathrm{~mm}$} & \\
\hline Slice thickness & \multicolumn{3}{|c|}{$>4 \mathrm{~mm}$} & \\
\hline Coverage & \multicolumn{3}{|c|}{ Full kidney } & \\
\hline $\begin{array}{l}\text { Parallel imaging } \\
\text { factor }\end{array}$ & \multicolumn{3}{|c|}{2} & \\
\hline Fat suppression & \multicolumn{3}{|c|}{ SPAIR } & \\
\hline TR (s) & \multicolumn{3}{|c|}{4} & \\
\hline $\mathrm{TE}(\mathrm{ms})$ & \multicolumn{3}{|c|}{$\operatorname{Min}(<\mathbf{1 0 0})$} & \\
\hline Averages & \multicolumn{3}{|c|}{3} & \\
\hline Breathing mode & \multicolumn{3}{|c|}{$\begin{array}{c}\text { Respiratory gated } \\
\text { (or free breathing with post-hoc motion correction) }\end{array}$} & \\
\hline Cardiac gating & \multicolumn{3}{|c|}{ no } & \\
\hline Diffusion gradients & \multicolumn{3}{|c|}{ Monopolar } & \\
\hline & $\mathrm{ADC}$ & IVIM & DTI & \\
\hline \# b-values & 4 & $>6$ & $>2$ & \\
\hline Suggested b-values & $0,100,200,800$ & $0,30,70,100,200,400,800$ & $0,200,800$ & \\
\hline \# directions & 3 & 3 & 12 or more & \\
\hline Time (min) & 2 & 3.8 & 5 & \\
\hline $\begin{array}{l}\text { Distortion } \\
\text { correction }\end{array}$ & \multicolumn{3}{|c|}{ Recommended } & \\
\hline Registration & \multicolumn{3}{|c|}{ Recommended, unilateral if possible } & \\
\hline $\begin{array}{l}\text { Image quality } \\
\text { control }\end{array}$ & \multicolumn{3}{|c|}{ Recommended } & \\
\hline ROI placement & \multicolumn{3}{|c|}{$\mathrm{b}=0$ image } & \\
\hline Cortical ROI & \multicolumn{3}{|c|}{1 stripe / slice $;>3$ slices } & \\
\hline Medullary ROI & \multicolumn{3}{|c|}{3 samples / slice $;>3$ slices } & \\
\hline Reporting & \multicolumn{3}{|c|}{ Cortex and Medulla } & \\
\hline $\begin{array}{l}\text { Metric statistics } \\
\text { reporting }\end{array}$ & \multicolumn{3}{|c|}{ Mean, Median, Standard deviation, ROI size } & \\
\hline Diffusion units & \multicolumn{3}{|c|}{$10^{-3} \mathrm{~mm}^{2} / \mathrm{s}$} & \\
\hline Map format & \multicolumn{3}{|c|}{ Colormap, fused with anatomy if possible } & \\
\hline
\end{tabular}

Recommendations in bold are derived from consensus view of the expert panel. Weight of each recommendation is color coded (green $=$ consensus $(\geq 75 \%)$; orange $=$ preference $(\geq 60 \%)$

to enable reasonable comparison of analogous MRI biomarkers (e.g., ADC and MD) in future datasets. Taking all of this into account, we recommend the following $b$ value sets (Table 4): for monoexponential DWI studies, $b=0,100$, 200, $800 \mathrm{~s} / \mathrm{mm}^{2}, 3$ directions; for IVIM studies $b=0,30$, $70,100,200,400,800 \mathrm{~s} / \mathrm{mm}^{2}, 3$ directions; for DTI studies, $b=0,200,800 \mathrm{~s} / \mathrm{mm}^{2}, 12$ or more directions.

Manual ROI placement had consensus support over automatic (e.g., histogram-based) placement, with the unweighted $(b=0)$ image having consensus support for ROI prescription. Cortical ROIs should be continuous stripes (one per slice), unless structural abnormalities prevent this, while medullary ROIs should be separate, with three regions sampled (upper, middle, lower poles). Generally, all slices from whole kidney coverage should be sampled with the exception of the two outermost slices where region delineation may be unclear. The consensus support for manual ROI placement is also interesting given the recent trend for machine learning (ML) and artificial intelligence (AI) in the medicine. Some efforts were made recently to adopt these techniques to renal DWI, especially in the detection of early acute renal allograft rejection [90-93]. However, great care is needed when trying to translate these approaches into the clinical arena, particularly in terms of clinical validation and measured patient-centric outcomes. We are confident that these techniques will play an important part in subsequent 
research studies, influence clinical translation and constitute a major focus for discussion in future versions of these recommendations.

We acknowledge some limitations in the procedures used to generate recommendations in this work. First, all entries in the literature review were assigned equal weight irrespective of population size or technological availability. Heterogeneity also exists in the survey process, in which participant elections may have been driven by different priorities and informed by different levels of clinical or technical experience. In addition, while we modeled our approach on the Delphi consensus procedure, its application was adjusted for the purposes of this review and its timeframe. The survey also highlighted other areas of disagreement between the participants. In particular, it was not possible to obtain a consensus on technical questions like the use of segmented echo planar acquisitions, or the advantage of bipolar diffusion gradients. We have not included a strategy of noise correction (pure Rician or otherwise) for more accurate quantification, but practical approaches exist that may be amenable to broad guidelines in a future iteration [85]. We also acknowledge that the imaging gradient contributions to the nominally unweighted $(b=0)$ image might lead to a potential source of error, especially in assessing $f$ and $D^{*}$. The full effect of this source of error has yet to be evaluated in the kidney literature. This work summarizes the large evidence base for a nonzero perfusion fraction, but the next level of standardization might refine processing to take full $b$ matrices into account $[94,95]$.

We have also not issued a standardized prescription for phantom quality control, which has proven beneficial to DWI standardization efforts in other contexts [96, 97]; the choice of and agreement upon such a phantom for renal DWI can be revisited in the next standardization iteration. Uncertainty exists also for physiological questions such as the effect of diet on DWI. As some of these issues have already been partly addressed in the literature, the survey indicates that currently available evidence may not be sufficient for conclusive resolution. This report should, therefore, motivate a significant effort to investigate these dedicated methodological questions.

\section{Conclusions}

The present work has summarized trends in the literature of renal diffusion MRI to date and their correlation with aspects of protocol design to direct future research efforts in the field of renal DWI. In pursuit of minimizing interstudy and inter-site variation, for the generation of evidence basis for reliable and high impact of imaging markers for renal disease, and with the guidance of a Delphi-based consensus process of experts in the field, we have generated a set of recommendations for future data collection. The recommended protocols have been chosen to be achievable by any center with clinical MRI capabilities and enable future multicentre pooling of data when equivalent protocols have been used. Therefore, these recommendations should be taken into account when starting new studies in the field of renal DWI and when reviewing submitted work in this area. We expect this recommendation process to be an iterative one and ensuing efforts may refine or add to these recommendations. To allow both growth and innovation in the field, as well as harmonization, "deviations" from these recommendations should be justified in the future studies and submissions for publication. There recommendations are intended to be updated when new evidence from ongoing or future studies is made available and change any of the recommended parameters.

Importantly, these translational efforts do not replace and are not in conflict with ongoing innovation efforts to uncover more specific biomarkers from renal DWI with more advanced methods. Instead, they reflect a view that commitment toward producing generalizable workflows in parallel will yield tremendous benefits to the field as a whole and increase chances of clinical impact on a larger scale.

Author contributions AL, AC, CL, SF, IAM, OB, FN, KS, AP, IAD, J-PV, KD, MN, RPL, SP, SDS, JP, ZJW, MF, HCT, PPr, MS, TN, PPu, SS and EES: contributing technical details of protocols or answering surveys. AL, AC, IAM, PPu and EES: helping to collect, tabulate and compare protocols and surveys submitted. AL and EES: helping to develop, organize, and document the online supplementary material (github or equivalent). AL, AC, CL, IAM, KS, HCT, TN, PPu and EES: regularly taking part in teleconferences or discussions of the panel to define a consensus. AL, IAM, SS and EES: other substantial contributions to the conception or design of the work; or the acquisition, analysis, or interpretation of data for the work (please specify). AL, IAM, J-PV, HCT, MS, TN and EES: drafting the work. AL, AC, CL, SF, IAM, OB, FN, KS, AP, IAD, J-PV, KD, MN, RPL, SP, SDS, JP, ZJW, MF, HCT, PPr, MS, TN, PPu, SS and EES: revising the work critically for important intellectual content. AL, AC, CL, SF, IAM, OB, FN, KS, AP, IAD, J-PV, KD, MN, RPL, SP, SDS, JP, ZJW, MF, HCT, PPr, MS, TN, PPu, SS and EES: final approval of the version to be published. AL, AC, CL, SF, IAM, OB, FN, KS, AP, IAD, J-PV, KD, MN, RPL, SP, SDS, JP, ZJW, MF, PPr, MS, TN, PPu, SS and EES: agreement to be accountable for all aspects of the work in ensuring that questions related to the accuracy or integrity of any part of the work are appropriately investigated and resolved.

Funding The article is based upon work from COST Action Magnetic Resonance Imaging Biomarkers for Chronic Kidney Disease (PARENCHIMA, COST Action CA16103, www.renalmri.org), funded by COST (European Cooperation in Science and Technology), www. cost.eu. For additional information please visit PARENCHIMA project website: www.renalmri.org. MIA funding from the Medical Research Council (Grant No. MR/R02264X/1). BO Grant support (2016-2018) from NIH NIDDK individual fellowship 1F32DK109591. SK was supported by the Biomarker Enterprise to Attack Diabetic Kidney Disease project funded by the Innovative Medicines Initiative 2 Joint Undertaking under Grant agreement 115974. This joint undertaking received 
support from the European Union's Horizon 2020 Research and Innovation programme and European Federation of Pharmaceutical Industries and Associations. PA receives funding from the German Research Council (DFG; Collaborative Research Centre SFB 1365 Renoprotection). J-PV and THC are supported by the Swiss National Science foundation Nb: IZCOZ0_177140/1. DK, NM, SEE research collaboration with Siemens. LRP Grant funding from Boehringer-Ingelheim. PJ receives funding from the German Ministry for Education and Research (BMBF; Grant VIP 03P00081). NT is supported by Germany Research Foundation, Collaborative Research Center 1365.

\section{Compliance with ethical standards}

Conflict of interest DK, NM, SEE research collaboration with Siemens. NT is founder and CEO of MRI TOOLS GmbH, Berlin, Germany. All other authors have no conflicts of interest and nothing to declare.

Ethical approval This article does not report any new studies with human participants performed by any of the authors.

Open Access This article is licensed under a Creative Commons Attribution 4.0 International License, which permits use, sharing, adaptation, distribution and reproduction in any medium or format, as long as you give appropriate credit to the original author(s) and the source, provide a link to the Creative Commons licence, and indicate if changes were made. The images or other third party material in this article are included in the article's Creative Commons licence, unless indicated otherwise in a credit line to the material. If material is not included in the article's Creative Commons licence and your intended use is not permitted by statutory regulation or exceeds the permitted use, you will need to obtain permission directly from the copyright holder. To view a copy of this licence, visit http://creativecommons.org/licenses/by/4.0/.

\section{References}

1. Chandarana H, Lee VS (2009) Renal functional MRI: are we ready for clinical application? AJR Am J Roentgenol 192(6):1550-1557

2. Grenier N, Basseau F, Ries M, Tyndal B, Jones R, Moonen C (2003) Functional MRI of the kidney. Abdom Imaging 28(2):164-175

3. Hueper K, Khalifa AA, Brasen JH, Vo Chieu VD, Gutberlet M, Wintterle S, Lehner F, Richter N, Peperhove M, Tewes S, Weber K, Haller H, Wacker F, Gwinner W, Gueler F, Hartung D (2016) Diffusion-Weighted imaging and diffusion tensor imaging detect delayed graft function and correlate with allograft fibrosis in patients early after kidney transplantation. J Magn Reson Imaging 44(1):112-121

4. Thoeny HC, De Keyzer F (2011) Diffusion-weighted MR imaging of native and transplanted kidneys. Radiology 259(1):25-38

5. Zhou JY, Wang YC, Zeng CH, Ju SH (2018) Renal functional MRI and its application. J Magn Reson Imaging 48(4):863-881

6. Ljimani A, Wittsack HJ, Lanzman RS (2018) Functional MRI in transplanted kidneys. Abdom Radiol (NY) 43(10):2615-2624

7. Malyarenko DI, Wilmes LJ, Arlinghaus LR, Jacobs MA, Huang W, Helmer KG, Taouli B, Yankeelov TE, Newitt D, Chenevert TL (2016) QIN DAWG validation of gradient nonlinearity bias correction workflow for quantitative diffusion-weighted imaging in multicenter trials. Tomography 2(4):396-405

8. Newitt DC, Zhang Z, Gibbs JE, Partridge SC, Chenevert TL, Rosen MA, Bolan PJ, Marques HS, Aliu S, Li W, Cimino L, Joe
BN, Umphrey H, Ojeda-Fournier H, Dogan B, Oh K, Abe H, Drukteinis J, Esserman LJ, Hylton NM, Team AT, Investigators IST (2019) Test-retest repeatability and reproducibility of ADC measures by breast DWI: results from the ACRIN 6698 trial. J Magn Reson Imaging 49(6):1617-1628

9. Shukla-Dave A, Obuchowski NA, Chenevert TL, Jambawalikar S, Schwartz LH, Malyarenko D, Huang W, Noworolski SM, Young RJ, Shiroishi MS, Kim H, Coolens C, Laue H, Chung C, Rosen M, Boss M, Jackson EF (2019) Quantitative imaging biomarkers alliance (QIBA) recommendations for improved precision of DWI and DCE-MRI derived biomarkers in multicenter oncology trials. J Magn Reson Imaging 49(7):e101-e121

10. Caroli A, Schneider M, Friedli I, Ljimani A, De Seigneux S, Boor P, Gullapudi L, Kazmi I, Mendichovszky IA, Notohamiprodjo M, Selby NM, Thoeny HC, Grenier N, Vallee JP (2018) Diffusionweighted magnetic resonance imaging to assess diffuse renal pathology: a systematic review and statement paper. Nephrol Dial Transplant 33(suppl_2):ii29-ii40

11. Lynn MR (1986) Determination and quantification of content validity. Nurs Res 35(6):382-385

12. Muller BG, van den Bos W, Brausi M, Cornud F, Gontero P, Kirkham A, Pinto PA, Polascik TJ, Rastinehad AR, de Reijke TM, de la Rosette JJ, Ukimura O, Villers A, Walz J, Wijkstra H, Marberger M (2014) Role of multiparametric magnetic resonance imaging (MRI) in focal therapy for prostate cancer: a Delphi consensus project. BJU Int 114(5):698-707

13. Taylor SA, Avni F, Cronin CG, Hoeffel C, Kim SH, Laghi A, Napolitano M, Petit P, Rimola J, Tolan DJ, Torkzad MR, Zappa M, Bhatnagar G, Puylaert CAJ, Stoker J (2017) The first joint ESGAR/ESPR consensus statement on the technical performance of cross-sectional small bowel and colonic imaging. Eur Radiol 27(6):2570-2582

14. Stejskal EO, Tanner JE (1965) Spin diffusion measurements: spin echoes in the presence of a time-dependent field gradient. J Chem Phys 42(1):288-292

15. Cheung JS, Fan SJ, Chow AM, Zhang J, Man K, Wu EX (2010) Diffusion tensor imaging of renal ischemia reperfusion injury in an experimental model. NMR Biomed 23(5):496-502

16. Zhang JL, Sigmund EE, Rusinek H, Chandarana H, Storey P, Chen Q, Lee VS (2012) Optimization of $b$ value sampling for diffusion-weighted imaging of the kidney. Magn Reson Med 67(1):89-97

17. Lemke A, Stieltjes B, Schad LR, Laun FB (2011) Toward an optimal distribution of $\mathrm{b}$ values for intravoxel incoherent motion imaging. Magn Reson Imaging 29(6):766-776

18. Le Bihan D, Breton E, Lallemand D, Grenier P, Cabanis E, LavalJeantet M (1986) MR imaging of intravoxel incoherent motions: application to diffusion and perfusion in neurologic disorders. Radiology 161(2):401-407

19. Muller MF, Prasad PV, Edelman RR (1998) Can the IVIM model be used for renal perfusion imaging? Eur J Radiol 26(3):297-303

20. Thoeny HC, Zumstein D, Simon-Zoula S, Eisenberger U, De Keyzer F, Hofmann L, Vock P, Boesch C, Frey FJ, Vermathen $P$ (2006) Functional evaluation of transplanted kidneys with diffusion-weighted and BOLD MR imaging: initial experience. Radiology 241(3):812-821

21. Ljimani A, Lanzman RS, Muller-Lutz A, Antoch G, Wittsack HJ (2017) Non-gaussian diffusion evaluation of the human kidney by Pade exponent model. J Magn Reson Imaging. https://doi. org/10.1002/jmri.25742

22. Blondin D, Lanzman RS, Mathys C, Grotemeyer D, Voiculescu A, Sandmann W, Rump LC, Modder U, Wittsack HJ (2009) Functional MRI of transplanted kidneys using diffusion-weighted imaging. RoFo: Fortschr Geb Rontgenstrahlen Nuklearmed 181(12):1162-1167 
23. Schneider MJ, Notohamiprodjo M, Dietrich O (2019) IVIM MRI in the Kidney. In: LeBihan D, Iima M, Federau C, Sigmund EE (eds) Intravoxel incoherent motion (IVIM) MRI, vol 1. Pan Stanford Publishing, Singapore, pp 261-282

24. Gurney-Champion OJ, Klaassen R, Froeling M, Barbieri S, Stoker J, Engelbrecht MRW, Wilmink JW, Besselink MG, Bel A, van Laarhoven HWM, Nederveen AJ (2018) Comparison of six fit algorithms for the intra-voxel incoherent motion model of diffusion-weighted magnetic resonance imaging data of pancreatic cancer patients. PLoS One 13(4):e0194590

25. Cho GY, Moy L, Zhang JL, Baete S, Lattanzi R, Moccaldi M, Babb JS, Kim S, Sodickson DK, Sigmund EE (2015) Comparison of fitting methods and $b$ value sampling strategies for intravoxel incoherent motion in breast cancer. Magn Reson Med 74(4):1077-1085

26. Meeus EM, Novak J, Withey SB, Zarinabad N, Dehghani H, Peet AC (2017) Evaluation of intravoxel incoherent motion fitting methods in low-perfused tissue. J Magn Reson Imaging 45(5):1325-1334

27. Jalnefjord O, Andersson M, Montelius M, Starck G, Elf AK, Johanson V, Svensson J, Ljungberg M (2018) Comparison of methods for estimation of the intravoxel incoherent motion (IVIM) diffusion coefficient $(D)$ and perfusion fraction $(f)$. MAGMA 31(6):715-723

28. Barbieri S, Donati OF, Froehlich JM, Thoeny HC (2016) Impact of the calculation algorithm on biexponential fitting of diffusionweighted MRI in upper abdominal organs. Magn Reson Med 75(5):2175-2184

29. van Baalen S, Leemans A, Dik P, Lilien MR, Ten Haken B, Froeling $M$ (2017) Intravoxel incoherent motion modeling in the kidneys: comparison of mono-, bi-, and triexponential fit. J Magn Reson Imaging 46(1):228-239

30. van der Bel R, Gurney-Champion OJ, Froeling M, Stroes ESG, Nederveen AJ, Krediet CTP (2017) A tri-exponential model for intravoxel incoherent motion analysis of the human kidney: in silico and during pharmacological renal perfusion modulation. Eur J Radiol 91:168-174

31. Ebrahimi B, Saad A, Jiang K, Ferguson CM, Tang H, Woollard JR, Glockner JF, Textor SC, Lerman LO (2017) Renal adiposity confounds quantitative assessment of markers of renal diffusion with MRI: a proposed correction method. Invest Radiol 52(11):672-679

32. Hilbert F, Bock M, Neubauer H, Veldhoen S, Wech T, Bley TA, Kostler H (2016) An intravoxel oriented flow model for diffusionweighted imaging of the kidney. NMR Biomed 29(10):1403-1413

33. Ye Q, Chen Z, Zhao Y, Zhang Z, Miao H, Xiao Q, Wang M, Li $J$ (2016) Initial experience of generalized intravoxel incoherent motion imaging and diffusion tensor imaging (GIVIM-DTI) in healthy subjects. J Magn Reson Imaging 44(3):732-738

34. Hagmann P, Jonasson L, Maeder P, Thiran JP, Wedeen VJ, Meuli $R$ (2006) Understanding diffusion MR imaging techniques: from scalar diffusion-weighted imaging to diffusion tensor imaging and beyond. Radiographics 26(Suppl 1):S205-S223

35. Basser PJ (1995) Inferring microstructural features and the physiological state of tissues from diffusion-weighted images. NMR Biomed 8(7-8):333-344

36. Jones DK, Basser PJ (2004) "Squashing peanuts and smashing pumpkins": how noise distorts diffusion-weighted MR data. Magn Reson Med 52(5):979-993

37. Blondin D, Lanzman RS, Klasen J, Scherer A, Miese F, Kropil P, Wittsack HJ (2011) Diffusion-attenuated MRI signal of renal allografts: comparison of two different statistical models. AJR Am J Roentgenol 196(6):W701-W705

38. Kataoka M, Kido A, Yamamoto A, Nakamoto Y, Koyama T, Isoda H, Maetani Y, Umeoka S, Tamai K, Saga T, Morisawa N, Mori S, Togashi K (2009) Diffusion tensor imaging of kidneys with respiratory triggering: optimization of parameters to demonstrate anisotropic structures on fraction anisotropy maps. J Magn Reson Imaging 29(3):736-744

39. Lu L, Sedor JR, Gulani V, Schelling JR, O'Brien A, Flask CA, MacRae Dell K (2011) Use of diffusion tensor MRI to identify early changes in diabetic nephropathy. Am J Nephrol 34(5):476-482

40. Notohamiprodjo M, Glaser C, Herrmann KA, Dietrich O, Attenberger UI, Reiser MF, Schoenberg SO, Michaely HJ (2008) Diffusion tensor imaging of the kidney with parallel imaging: initial clinical experience. Invest Radiol 43(10):677-685

41. Ries M, Jones RA, Basseau F, Moonen CT, Grenier N (2001) Diffusion tensor MRI of the human kidney. J Magn Reson Imaging 14(1):42-49

42. Sigmund EE, Vivier HV, Sui D, Lamparello N, Tantillo K, Mikheev A, Rusinek H, Babb J, Storey P, Lee VS, Chandarana H (2012) Intravoxel incoherent motion (IVIM) and diffusion tensor imaging (DTI) in renal tissue under hydration and furosemide flow challenges. Radiology 263(3):758-769

43. Thoeny HC, Binser T, Roth B, Kessler TM, Vermathen P (2009) Noninvasive assessment of acute ureteral obstruction with diffusion-weighted MR imaging: a prospective study. Radiology 252(3):721-728

44. Wu M, Lin Y, Shieh C, Wan Y, Yen TH, Ng K, Wai Y, Wang J (2011) Measuring anisotropic diffusion in kidney using MRI. Acad Radiol 18(9):1168-1174

45. Notohamiprodjo M, Dietrich O, Horger W, Horng A, Helck AD, Herrmann KA, Reiser MF, Glaser C (2010) Diffusion tensor imaging (DTI) of the kidney at 3 tesla-feasibility, protocol evaluation and comparison to 1.5 Tesla. Invest Radiol 45(5):245-254

46. Froeling M, Nederveen AJ, Nicolay K, Strijkers GJ (2013) DTI of human skeletal muscle: the effects of diffusion encoding parameters, signal-to-noise ratio and $\mathrm{T} 2$ on tensor indices and fiber tracts. NMR Biomed 26(11):1339-1352

47. Sulkowska K, Palczewski P, Duda-Zysk A, Szeszkowski W, Wojcik D, Kownacka-Piotrowska D, Golebiowski M (2015) Diffusion-weighted MRI of kidneys in healthy volunteers and living kidney donors. Clin Radiol 70(10):1122-1127

48. Binser T, Thoeny HC, Eisenberger U, Stemmer A, Boesch C, Vermathen $P$ (2010) Comparison of physiological triggering schemes for diffusion-weighted magnetic resonance imaging in kidneys. J Magn Reson Imaging 31(5):1144-1150

49. Donati OF, Chong D, Nanz D, Boss A, Froehlich JM, Andres E, Seifert B, Thoeny HC (2014) Diffusion-weighted MR imaging of upper abdominal organs: field strength and intervendor variability of apparent diffusion coefficients. Radiology 270(2):454-463

50. Eisenberger U, Binser T, Thoeny HC, Boesch C, Frey FJ, Vermathen $P$ (2014) Living renal allograft transplantation: diffusionweighted MR imaging in longitudinal follow-up of the donated and the remaining kidney. Radiology 270(3):800-808

51. Filli L, Wurnig M, Nanz D, Luechinger R, Kenkel D, Boss A (2014) Whole-body diffusion kurtosis imaging: initial experience on non-Gaussian diffusion in various organs. Invest Radiol 49(12):773-778

52. Friedli I, Crowe LA, Viallon M, Porter DA, Martin PY, de Seigneux S, Vallee JP (2015) Improvement of renal diffusionweighted magnetic resonance imaging with readout-segmented echo-planar imaging at 3 T. Magn Reson Imaging 33(6):701-708

53. Herrmann J, Wenzel U, Galler S, Schoennagel BP, Busch JD, Tozakidou M, Petersen KU, Joekel M, Bannas P, Yamamura J, Groth M, Adam G, Habermann CR (2017) Diffusion-weighted imaging of the kidneys in haemolytic uraemic syndrome. Eur Radiol. https://doi.org/10.1007/s00330-017-4848-2

54. Jerome NP, Orton MR, d'Arcy JA, Collins DJ, Koh DM, Leach MO (2014) Comparison of free-breathing with navigator-controlled acquisition regimes in abdominal diffusion-weighted 
magnetic resonance images: effect on ADC and IVIM statistics. J Magn Reson Imaging 39(1):235-240

55. Jin N, Deng J, Zhang L, Zhang Z, Lu G, Omary RA, Larson AC (2011) Targeted single-shot methods for diffusion-weighted imaging in the kidneys. J Magn Reson Imaging 33(6):1517-1525

56. Murtz P, Flacke S, Traber F, van den Brink JS, Gieseke J, Schild $\mathrm{HH}$ (2002) Abdomen: diffusion-weighted MR imaging with pulsetriggered single-shot sequences. Radiology 224(1):258-264

57. Ozcelik U, Cevik H, Bircan HY, Yarbug Karakayali F, Isiklar I, Haberal M (2017) Evaluation of transplanted kidneys and comparison with healthy volunteers and kidney donors with diffusionweighted magnetic resonance imaging: initial experience. Exp Clin Transplant. https://doi.org/10.6002/ect.2016.0341

58. Siegel CL, Aisen AM, Ellis JH, Londy F, Chenevert TL (1995) Feasibility of MR diffusion studies in the kidney. J Magn Reson Imaging 5(5):617-620

59. Wang WJ, Pui MH, Guo Y, Wang LQ, Wang HJ, Liu M (2014) 3T magnetic resonance diffusion tensor imaging in chronic kidney disease. Abdom Imaging 39(4):770-775

60. Zhao J, Wang ZJ, Liu M, Zhu J, Zhang X, Zhang T, Li S, Li Y (2014) Assessment of renal fibrosis in chronic kidney disease using diffusion-weighted MRI. Clin Radiol 69(11):1117-1122

61. Zheng Z, Yan T, Jia J, Li D, Wei L, Shang W, Shi H (2018) Assessment of renal pathological changes in lupus nephritis using diffusion weighted imaging: a multiple correspondence analysis. Kidney Blood Press Res 43(3):847-859

62. Wang WJ, Pui MH, Guo Y, Hu XS, Wang HJ, Yang D (2014) MR diffusion tensor imaging of normal kidneys. J Magn Reson Imaging 40(5):1099-1102

63. Chuck NC, Steidle G, Blume I, Fischer MA, Nanz D, Boss A (2013) Diffusion tensor imaging of the kidneys: influence of $b$ value and number of encoding directions on image quality and diffusion tensor parameters. J Clin Imaging Sci 3:53

64. Chan RW, Von Deuster C, Stoeck CT, Harmer J, Punwani S, Ramachandran N, Kozerke S, Atkinson D (2014) High-resolution diffusion tensor imaging of the human kidneys using a free-breathing, multi-slice, targeted field of view approach. NMR Biomed 27(11):1300-1312

65. Cutajar M, Clayden JD, Clark CA, Gordon I (2011) Test-retest reliability and repeatability of renal diffusion tensor MRI in healthy subjects. Eur J Radiol 80(3):e263-e268

66. Zheng Z, Shi H, Zhang J, Zhang Y (2014) Renal water molecular diffusion characteristics in healthy native kidneys: assessment with diffusion tensor MR imaging. PLoS One 9(12):e113469

67. Kido A, Kataoka M, Yamamoto A, Nakamoto Y, Umeoka S, Koyama T, Maetani Y, Isoda H, Tamai K, Morisawa N, Saga T, Mori S, Togashi K (2010) Diffusion tensor MRI of the kidney at 3.0 and 1.5 Tesla. Acta Radiol (Stockholm, Sweden : 1987) 51(9):1059-1063

68. Seif M, Lu H, Boesch C, Reyes M, Vermathen P (2015) Image registration for triggered and non-triggered DTI of the human kidney: reduced variability of diffusion parameter estimation. J Magn Reson Imaging 41(5):1228-1235

69. Gurses B, Kilickesmez O, Tasdelen N, Firat Z, Gurmen N (2011) Diffusion tensor imaging of the kidney at 3 Tesla MRI: normative values and repeatability of measurements in healthy volunteers. Diagn Interv Radiol (Ank, Turk) 17(4):317-322

70. Lupica R, Mormina E, Lacquaniti A, Trimboli D, Bianchimano B, Marino S, Bramanti P, Longo M, Buemi M, Granata F (2016) 3 tesla-diffusion tensor imaging in autosomal dominant polycystic kidney disease: the nephrologist's point of view. Nephron 134(2):73-80

71. Liu AL, Mikheev A, Rusinek H, Huang WC, Wysock JS, Babb JS, Feiweier T, Stoffel D, Chandarana H, Sigmund EE (2018) REnal flow and microstructure anisotropy (REFMAP) MRI in normal and peritumoral renal tissue. J Magn Reson Imaging 48(1):188-197
72. Barbieri S, Donati OF, Froehlich JM, Thoeny HC (2016) Comparison of intravoxel incoherent motion parameters across MR imagers and field strengths: evaluation in upper abdominal organs. Radiology 279(3):784-794

73. Deux JF, Audard V, Brugieres P, Habibi A, Manea EM, GuillaudDanis C, Godeau B, Galacteros F, Stehle T, Lang P, Grimbert P, Audureau E, Rahmouni A, Bartolucci P (2017) Magnetic resonance imaging assessment of kidney oxygenation and perfusion during sickle cell vaso-occlusive crises. Am J Kidney Dis 69(1):51-59

74. Filli L, Wurnig MC, Luechinger R, Eberhardt C, Guggenberger R, Boss A (2015) Whole-body intravoxel incoherent motion imaging. Eur Radiol 25(7):2049-2058

75. Friedli I, Crowe LA, de Perrot T, Berchtold L, Martin PY, de Seigneux S, Vallee JP (2017) Comparison of readout-segmented and conventional single-shot for echo-planar diffusion-weighted imaging in the assessment of kidney interstitial fibrosis. J Magn Reson Imaging. https://doi.org/10.1002/jmri.25687

76. Notohamiprodjo M, Chandarana H, Mikheev A, Rusinek H, Grinstead J, Feiweier T, Raya JG, Lee VS, Sigmund EE (2015) Combined intravoxel incoherent motion and diffusion tensor imaging of renal diffusion and flow anisotropy. Magn Reson Med 73(4): 1526-1532

77. Wurnig MC, Donati OF, Ulbrich E, Filli L, Kenkel D, Thoeny HC, Boss A (2015) Systematic analysis of the intravoxel incoherent motion threshold separating perfusion and diffusion effects: proposal of a standardized algorithm. Magn Reson Med 74(5):1414-1422

78. Lv J, Huang W, Zhang J, Wang X (2018) Performance of U-net based pyramidal lucas-kanade registration on free-breathing multi- $b$-value diffusion MRI of the kidney. Br J Radiol 91(1086):20170813

79. de Bazelaire CM, Duhamel GD, Rofsky NM, Alsop DC (2004) MR imaging relaxation times of abdominal and pelvic tissues measured in vivo at 3.0 T: preliminary results. Radiology 230(3):652-659

80. Norman Dalkey OH (1963) An experimental application of the Delphi method to the use of experts. Manag Sci 9(3):458-467

81. Jones D (2004) The effect of gradient sampling schemes on measures derived from diffusion tensor MRI: a Monte Carlo study. Magn Reson Med 51(4):807-815

82. Meeus EM, Novak J, Dehghani H, Peet AC (2018) Rapid measurement of intravoxel incoherent motion (IVIM) derived perfusion fraction for clinical magnetic resonance imaging. MAGMA 31(2):269-283

83. While PT (2017) A comparative simulation study of bayesian fitting approaches to intravoxel incoherent motion modeling in diffusionweighted MRI. Magn Reson Med 78(6):2373-2387

84. Coupe P, Manjon JV, Gedamu E, Arnold D, Robles M, Collins DL (2010) Robust Rician noise estimation for MR images. Med Image Anal 14(4):483-493

85. Reeder SB, Wintersperger BJ, Dietrich O, Lanz T, Greiser A, Reiser MF, Glazer GM, Schoenberg SO (2005) Practical approaches to the evaluation of signal-to-noise ratio performance with parallel imaging: application with cardiac imaging and a 32-channel cardiac coil. Magn Reson Med 54(3):748-754

86. Stanisz GJ, Odrobina EE, Pun J, Escaravage M, Graham SJ, Bronskill MJ, Henkelman RM (2005) T1, T2 relaxation and magnetization transfer in tissue at 3T. Magn Reson Med 54(3):507-512

87. Alanen A, Nummi P, Kormano M, Irjala K (1987) Proton T1 relaxation time of normal and abnormal urine. Acta Radiol (Stockh, Swed 1987) 28(5):601-602

88. Azoury R, Abrashkin S, Weininger J, Iaina A (1988) Proton MR properties of lyophilized urine samples from normal and stone former individuals. Magn Reson Imaging 6(1):49-52

89. Brown JJ, vanSonnenberg E, Gerber KH, Strich G, Wittich GR, Slutsky RA (1985) Magnetic resonance relaxation times of 
percutaneously obtained normal and abnormal body fluids. Radiology 154(3):727-731

90. Abdeltawab H, Shehata M, Shalaby A, Khalifa F, Mahmoud A, ElGhar MA, Dwyer AC, Ghazal M, Hajjdiab H, Keynton R, El-Baz A (2019) A novel CNN-based CAD system for early assessment of transplanted kidney dysfunction. Sci Rep 9(1):5948

91. Hollis E, Shehata M, Abou El-Ghar M, Ghazal M, El-Diasty T, Merchant M, Switala AE, El-Baz A (2017) Statistical analysis of ADCs and clinical biomarkers in detecting acute renal transplant rejection. Br J Radiol 90(1080):20170125

92. Shehata M, Mahmoud A, Soliman A, Khalifa F, Ghazal M, ElGhar MA, El-Melegy M, El-Baz A (2018) 3D kidney segmentation from abdominal diffusion MRI using an appearance-guided deformable boundary. PLoS One 13:1-21

93. Shehata M, Khalifa F, Soliman A, Ghazal M, Taher F, El-Ghar MA, Dwyer AC, Farb G, Keyton RS, El-Baz A (2018) Computer-aided diagnostic system for early detection of acute renal transplant rejection using diffusion-weighted MRI. Trans Biomed Eng 66:1-14

94. Basser PJ, Mattiello J, LeBihan D (1994) Estimation of the effective self-diffusion tensor from the NMR spin echo. J Magn Reson B 103(3):247-254
95. Mattiello J, Basser PJ, Le Bihan D (1997) The $b$ matrix in diffusion tensor echo-planar imaging. Magn Reson Med 37(2):292-300

96. Malyarenko D, Galban CJ, Londy FJ, Meyer CR, Johnson TD, Rehemtulla A, Ross BD, Chenevert TL (2013) Multi-system repeatability and reproducibility of apparent diffusion coefficient measurement using an ice-water phantom. J Magn Reson Imaging 37(5):1238-1246

97. Keenan KE, Ainslie M, Barker AJ, Boss MA, Cecil KM, Charles C, Chenevert TL, Clarke L, Evelhoch JL, Finn P, Gembris D, Gunter JL, Hill DLG, Jack CR Jr, Jackson EF, Liu G, Russek SE, Sharma SD, Steckner M, Stupic KF, Trzasko JD, Yuan C, Zheng J (2018) Quantitative magnetic resonance imaging phantoms: a review and the need for a system phantom. Magn Reson Med 79(1):48-61

Publisher's Note Springer Nature remains neutral with regard to jurisdictional claims in published maps and institutional affiliations.

\section{Affiliations}

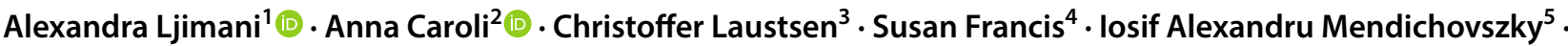 Octavia Bane $^{6} \cdot$ Fabio Nery $^{7} \cdot$ Kanishka Sharma $^{8} \cdot$ Andreas Pohlmann $^{9} \cdot$ Ilona A. Dekkers ${ }^{10}$. Jean-Paul Vallee ${ }^{11}$. Katja Derlin ${ }^{12}$. Mike Notohamiprodjo ${ }^{13,26} \cdot$ Ruth P. Lim $^{14}$. Stefano Palmucci ${ }^{15}$. Suraj D. Serai ${ }^{16}$. Joao Periquito ${ }^{9}$ Zhen Jane Wang ${ }^{17} \cdot$ Martijn Froeling ${ }^{18}$ (D) Harriet C. Thoeny ${ }^{19}$. Pottumarthi Prasad ${ }^{20} \cdot$ Moritz Schneider $^{21,22}$. Thoralf Niendorf $^{9} \cdot$ Pim Pullens $s^{23,24} \cdot$ Steven Sourbron ${ }^{8} \cdot$ Eric E. Sigmund ${ }^{25}$}

1 Department of Diagnostic and Interventional Radiology, Medical Faculty, University Dusseldorf, Moorenstr. 5, 40225 Düsseldorf, Germany

2 Department of Biomedical Engineering, Istituto di Ricerche Farmacologiche Mario Negri IRCCS, Bergamo, Italy

3 MR Research Centre, Department of Clinical Medicine, Aarhus University, Aarhus, Denmark

4 Sir Peter Mansfield Imaging Centre, University Park, University of Nottingham, Nottingham NG7 2RD, UK

5 Department of Radiology, Cambridge University Hospitals NHS Foundation Trust, Addenbrooke's Hospital, Cambridge, UK

6 Translational and Molecular Imaging Institute and Department of Radiology, Icahn School of Medicine at Mount Sinai, New York, NY, USA

7 Developmental Imaging and Biophysics Section, UCL Great Ormond Street Institute of Child Health, London, UK

8 Imaging Biomarkers Group, Department of Biomedical Imaging Sciences, University of Leeds, Leeds, UK

9 Berlin Ultrahigh Field Facility (B.U.F.F.), Max Delbrueck Center for Molecular Medicine in the Helmholtz Association, 13125 Berlin, Germany

10 Department of Radiology, Leiden University Medical Center, Leiden, The Netherlands

11 Department of Diagnostic, Geneva University Hospital and University of Geneva, 1211 Geneva-14, Switzerland
12 Department of Radiology, Hannover Medical School, Hannover, Germany

13 Die Radiologie, Munich, Germany

14 Department of Radiology, Austin Health, The University of Melbourne, Melbourne, Australia

15 Department of Medical Surgical Sciences and Advanced Technologies, Radiology I Unit, University Hospital "Policlinico-Vittorio Emanuele", University of Catania, Catania, Italy

16 Department of Radiology, Children's Hospital of Philadelphia, Philadelphia, PA, USA

17 Department of Radiology and Biomedical Imaging, University of California San Francisco, San Francisco, CA, USA

18 Department of Radiology, University Medical Center Utrecht, Utrecht, The Netherlands

19 Department of Radiology, Hôpital Cantonal Fribourgois (HFR), University of Fribourg, 1708 Fribourg, Switzerland

20 Department of Radiology, Center for Advanced Imaging, NorthShore University Health System, Evanston, IL, USA

21 Department of Radiology, University Hospital, LMU Munich, Munich, Germany

22 Comprehensive Pneumology Center, German Center for Lung Research, Munich, Germany

23 Ghent Institute for Functional and Metabolic Imaging, Ghent University, Ghent, Belgium 
24 Department of Radiology, University Hospital Ghent, Ghent, Belgium

25 Department of Radiology, Center for Biomedical Imaging (CBI), Center for Advanced Imaging Innovation and Research (CAI2R), NYU Langone Health, New York, NY, USA

26 Department of Radiology, University Hospital Tuebingen, Tübingen, Germany 